\title{
A CRITICAL REVISITING OF FINITE ELASTO-PLASTICITY
}

\author{
ELISA DAVOLI AND GILLES A. FRANCFORT
}

\begin{abstract}
We propose a new evolution model for large strain elastoplasticity. In a quasi-static setting the suggested model can be formulated as a variational evolution. We establish existence of a closely related evolution in a regularized context. We then discuss how the proposed model performs in both a rigid-plastic, and a one-dimensional settings and compare the results with those that could be achieved using other formulations.
\end{abstract}

\section{INTRODUCTION}

Deep drawing, rolling, and other metal forming processes cannot be apprehended within the framework of classical (small strain) elasto-plasticity because both large strains and large rotations are inherent to such processes. Finite plasticity should be the macroscopic answer. Unfortunately, more than 50 years after its inception, the field is still a "work in progress".

The 70's and 80's witnessed a flurry of competing theories. The controversies were numerous, ranging from the nature of the plastic strain to that of the yield criterium. Those are wonderfully recalled in [35]. The resulting mechanical strife subsequently waned without proper adjudication as the protagonists ceased their professional activities. Of course, finite plasticity is still an active field and new monographs on that topic are being published yearly. However, it seems difficult to extract a common thread among the various contributions; see for example the vastly different accounts of the topic in [3] versus [23].

With such an heritage, it would undoubtedly be preposterous on our part to aspire to an all encompassing vision of the current state of finite plasticity. Nor do we wish to resuscitate the battles of yore that pitted e.g. proponents of the Jaumann stress rate against those of the convected stress rate (see e.g. [23]), or still to weigh in on the thermodynamical correctness of such or such intermediate configuration....

Rather, we propose in this contribution to try and circumscribe a theory of finite plasticity with two guiding principles. On the one hand it should not overstep the fairly consensual confines of rational thermodynamics that were first elaborated by C. Truesdell and many equally famous co-workers [38]; for our purpose those famous contributions should be refined with the import of the theory of Standard Generalized Materials introduced by B. Halphen \& Q.S. NGUYen [22] because that approach draws its inspiration in convex duality, a very fruitful tool when dealing with the modeling of any kind of plastic behavior.

On the other hand the resulting model should accommodate the more recent advances in the modeling of rate independent evolutions within a variational framework. The theory of variational rate independent evolutions has in the past 20 
years or so proved to be a handy tool in the mathematical understanding of a variety of rate independent evolutions, be it brittle fracture [18], [12], [17], [11], various approaches to damage [19], [15], [28], or still small strain elasto-plasticity [9], etc..... To our knowledge the only mature attempt in the direction of finite strain elasto-plasticity is that of A. MiELKE, together with several co-workers; among the many contributions see in particular [30], [31], [26]. The resulting model will be commented upon and distinguished from ours in Remark 4.9 below.

Our goal will be achieved in Section 4 and, specifically, in Proposition 4.1 and Definition 4.6. To do so we will first establish a short list of guidelines in Section 2 , then use the full force of thermodynamical modeling - restricted to an isothermal setting - in Section 3. This will result in a coupled system of P.D.E.'s and O.D.E.'s (see Model 3.3) which, when specialized to a quasi-static setting, can be re-formulated as a variational evolution.

Unfortunately, the resulting variational model is intractable at present in its full generality. The formidable hurdles that need to be overcome will be further elaborated upon at the end of Section 4.

An adequate regularization of the model, similar in spirit to that performed in [26], alleviates all obstacles and results in a well-posed variational evolution. To this effect, one should introduce an additional energy which depends super-linearly on both the plastic strain and its gradient; in essence this amounts to introducing both hardening and gradient plasticity into the model. See [13], [14] for a model of gradient plasticity and e.g. [24], [16] for models of hardening. This is the object of Section 5 and, in particular of Theorem 5.5 where the existence of a variational evolution is established.

As of yet, we are unable to assert any kind of time regularity of the plastic strains associated with the evolution, and this in spite of the various regularizations. Consequently, we are also unable to prove that the regularized evolution is also a "classical" solution, that is that it satisfies a regularized flow rule; see by contrast $[16$, Section 4$]$ in the small strain setting.

The last two sections of the paper are an exercise in self-criticism. In Section 6 we specialize the obtained model to the rigid-plastic case. There, elastic deformations are constrained to be pure rotations. It is our unsubstantiated belief that a "good" elasto-plastic model should behave reasonably decently when specialized to that setting. Unfortunately, such may not be the case at least in the context of globally minimizing variational evolutions as illustrated in Lemma 6.1. That result seems to intimate that the relaxation (in the sense of the Calculus of Variations) of the obtained model is identically 0 , which does not augur well for a similar relaxation of the full elasto-plastic model. This should be mitigated by the realization that it is not so obvious that Lemma 6.1 actually computes a relevant quantity from the standpoint of relaxation because we have no clear picture of the correct functional framework. Further, this defect is not specific to our model, but it should equally affect all elasto-plastic models which abide by any kind of multiplicative decomposition of the deformation gradient. So the culprit, if there is one at all, is the multiplicative decomposition.....

This is confirmed in Section 7 where we demonstrate that any multiplicative model, when specialized to a one-dimensional setting, will produce unstable homogeneous solutions during any kind of loading process. Of course, once again, the notion of stability is tied to the consideration of some kind of energy functional 
which should lose positivity in some direction. Our choice of energy is dictated by our variational "attitude", but it might prove to be the wrong one.

Notationwise, $\mathbb{R}_{1}^{3}:=\left\{(x, y, z) \in \mathbb{R}_{+}^{*} \times \mathbb{R}_{+}^{*} \times \mathbb{R}_{+}^{*}: x y z=1\right\}$ and $1_{3}:=(1,1,1)$. Throughout, we identify unimodular diagonal $3 \times 3$-matrices with positive entries with elements of $\mathbb{R}_{1}^{3}$ with the understanding that the rules of matrix multiplication apply for an element of $\mathbb{R}_{1}^{3}$ multiplied by a $3 \times 3$-matrix.

The matrix inner product is the Frobenius product $\left.\left(A \cdot B=\operatorname{tr} B^{T} A\right)\right)$. Any $N \times N$-matrix $M$ (an element of $\mathbb{M}^{N \times N}$ ) will be decomposed, if needed, into $M=$ $\operatorname{tr}(M / N) I+M_{D}$ where $I$ is the identity matrix and $M_{D}$ stands for the deviatoric (trace free) part of $M$. The space of all symmetric $N \times N$-matrices is denoted by $\mathbb{M}_{s}^{N \times N}$ while the subspace of trace free such matrices is denoted by $\mathbb{M}_{D}^{N \times N}$. Also, we denote by $\mathbb{M}_{+}^{N \times N}$ the subset of $\mathbb{M}^{N \times N}$ of matrices with positive determinant.

First order time derivatives are denoted with a dot $(\dot{\alpha}:=d \alpha / d t)$ while the differential of a scalar-valued function $\mathcal{W}: \mathbb{R}^{P} \rightarrow \mathbb{R}$ is denoted by $\mathbf{D} \mathcal{W}$.

If $\mathbf{K}$ is a closed convex subset of $\mathbb{R}^{P}$, we denote, for any $k \in \mathbf{K}$, by $N_{\mathbf{K}}(k)$ the normal cone to $\mathbf{K}$ at $k$, that is

$$
N_{\mathbf{K}}(k):= \begin{cases}0, & k \in \operatorname{int} \mathbf{K} \\ \nu \in \mathbb{R}^{P} \text { s.t. } \nu \cdot\left(k^{\prime}-k\right) \leq 0 \forall k^{\prime} \in \mathbf{K}, & k \in \partial \mathbf{K} .\end{cases}
$$

We will always write $\|v\|_{L_{p}}$ for the $L_{p}$-norm of a field $v$, whatever the target space of that field might be: $\mathbb{R}^{n}, \mathbb{M}_{D}^{N \times N}, \ldots$. Also, for any finite dimensional space $X, \mathcal{M}_{b}(\Omega ; X)$ will denote the space of $X$-valued bounded Radon measures on $\Omega$.

Throughout, we will call modulus of continuity any monotonically increasing continuous function $\omega: \mathbb{R}_{+} \rightarrow \mathbb{R}$ with $\omega(0)=0$.

Finally $C$ will denote a generic positive constant, so that e.g. $2 C$ is written $C$.

\section{A SHORT LIST OF NECESSARY INGREDIENTS}

We propose in this short section to outline a short list of simple (simplistic?) principles that any elasto-plastic model should in our opinion conform to.

The widespread belief that elastic and plastic deformations should be composed translates at the deformation gradient level into a multiplicative decomposition of the corresponding strains. Of course popular consensus has never been an adequate measure of scientific correctness and we will illustrate in Sections 6,7 the thorny issues raised by such a decomposition. For now, we do abide by plastic tradition and assume a multiplicative decomposition of the deformation gradient (denoted henceforth by $F$ ) into an elastic part (denoted henceforth by $E$ ) and a plastic part (denoted henceforth by $P$ ) as manifestation of the composition, in no particular order, of the elastic and plastic deformations.

First and foremost common sense dictates that, in the absence of any kind of plastic deformation, finite elasto-plastic behavior should be purely elastic. If so, then the deformation should be such that $F=E$ whenever $P=I$. Since classical hyper-elasticity is based on the consideration of an energy

$$
\mathcal{W}: \mathbb{M}^{N \times N} \rightarrow \overline{\mathbb{R}}
$$

which only depends on $F$, then one should view $E$ as being endowed with the same geometric properties as $F$, i.e., as a linear mapping from the tangent space of the undeformed configuration into that of the deformed configuration. Consequently, either $F=E P$ in which case $P$ is a linear mapping from the tangent space of 
the undeformed configuration into itself, or $F=P E$, in which case $P$ is a linear mapping from the tangent space of the deformed configuration into itself. In both cases, $F$ should preserve orientation, and, in the absence of plastic deformation, so should $E$, so that we may as well assume that $\operatorname{det} P$ and $\operatorname{det} E>0$.

In either situation the free energy density of the system - in an isothermal setting - should be of the form $\mathcal{W}(E)$; it should also respect material frame indifference, that is

$$
\mathcal{W}(E)=\hat{\mathcal{W}}\left(E^{T} E\right) \text { where } \hat{\mathcal{W}}: \mathbb{M}_{s}^{N \times N} \rightarrow \overline{\mathbb{R}} .
$$

We will further assume henceforth that $\mathcal{W}$ and $\hat{\mathcal{W}}$ are continuous and $C^{1}$ on their domain.

Then, the advent of plasticity should be conditioned by a threshold on the (symmetric) Cauchy stress (henceforth denoted by $C$ ) because it is the stress that is directly measurable. So classically, one should impose that $C \in \mathbf{K}$, where $\mathbf{K}$ is a convex (possibly compact) subset of $\mathbb{M}_{s}^{N \times N}$ with $0 \in \mathbf{K}$.

If so, then one should strive to write the mechanical dissipation (denoted henceforth by $\mathscr{D}$ ) in the form

$$
\mathscr{D}=C \cdot T
$$

for some quantity $T$. According to Clausius-Duhem's inequality, $\mathscr{D} \geq 0$, at least in the absence of thermal dissipation. (This is a mere re-statement of the second law of thermodynamics.) If we adopt, as is commonly done in plasticity, the normality rule, then, whatever $T$ turns out to be, the resulting flow rule should be

$$
T \in N_{\mathbf{K}}(C)
$$

or, equivalently, its Biot form

$$
C \in \mathcal{H}(R) \text { with } \mathcal{H}(T):=\sup \left\{T \cdot C^{\prime}: C^{\prime} \in \mathbf{K}\right\} .
$$

Also, if metals or crystalline solids are being primarily investigated, then the plastic deformation should be isochoric throughout the evolution, hence the constraint

$$
\operatorname{det} P=1
$$

should be satisfied at all times.

Now we remark that, since by (2.4) $\operatorname{det} P>0$, the polar decomposition permits to decompose $P$ as

$$
P=Q P^{\prime} Q^{T} R
$$

or, equivalently as

$$
P=\bar{R} \bar{Q} P^{\prime \prime} \bar{Q}^{T},
$$

where $R, Q, \bar{R}, \bar{Q} \in S O(N)$ and $P^{\prime}, P^{\prime \prime}$ are diagonal matrices with positive entries in some preset orthonormal basis $\left\{\vec{e}_{i}: i=1, \ldots, N\right\}$. So, setting $R^{\prime}:=Q^{T} R, Q^{\prime}:=\bar{R} \bar{Q}$, the decomposition $F=E P$ also reads as

$$
F=E Q P^{\prime} R^{\prime}=E Q^{\prime} P^{\prime \prime} \bar{Q}^{T}
$$

while the decomposition $F=P E$ also reads as

$$
F=Q P^{\prime} R^{\prime} E=Q^{\prime} P^{\prime \prime} \bar{Q}^{T} E .
$$

Because of frame indifference, $\mathcal{W}\left(\bar{Q}^{T} E\right)=\mathcal{W}\left(R^{\prime} E\right)=\mathcal{W}(E)$, so that the decomposition $F=P E$ can read, with no prejudice, as

$$
F=Q P^{\prime} E, \text { with } P^{\prime} \text { diagonal. }
$$


Such is not the case for the decomposition $F=E P$ because $\mathcal{W}(E Q)=\mathcal{W}\left(E Q^{\prime}\right)=$ $\mathcal{W}(E)$ only when $\mathcal{W}$ is isotropic.

In conclusion, in the absence of isotropy, we are led to considering the following decomposition of the deformation gradient $F$

$$
F=Q P E, \text { with }\left\{\begin{array}{l}
Q \in S O(3) \\
P \text { diagonal with entries }>0 \\
\operatorname{det} P=1 \\
E \text { with } \operatorname{det} E>0 .
\end{array}\right.
$$

This is the decomposition that we advocate in the next section. As seen from the above developed argument the reverse multiplicative decomposition (in the terminology of [23, Section 11.19]) is favored by the polar decomposition. This decomposition is undoubtedly less frequently encountered in the classical literature on finite plasticity.

Remark 2.1. In the setting of crystal plasticity, the plastic deformation is usually restricted to a finite number of allowed slip directions, resulting in a model where the decomposition is indeed $F=E P$, with $P$ of the form $I+\sum_{i \in I} \gamma_{i} s_{i} \otimes m_{i}$ where $I$ is finite and $\left|s_{i}\right|=\left|m_{i}\right|=1, s_{i} \cdot m_{i}=0, i \in I$. This kind of model is outside our purview.

We will see in the next section the impact of decomposition (2.5) and observe in Remark 3.2 that there is no benefit in adopting the "classical" multiplicative decomposition even in an isotropic setting.

\section{A RAtional MODEL FOR ElASto-Plastic EVOlUtion}

As motivated in Section 2 above, the deformation gradient is decomposed as in (2.5). The resulting decomposition immediately suggests that both $P$ and $Q$ be viewed as internal variables.

We now engage in the classical thermo-mechanical derivation of the ensuing model with, as is customary in such derivations, no regard for the regularity of the fields. In other words, we assume that all fields have sufficient smoothness so that all required differentiations and changes of variables are licit. We refer to e.g. [21], [27] for a detailed exposition of the fundamentals of that derivation.

First we should rewrite $\mathcal{W}$ as a function all variables. Accordingly, define

$$
\mathcal{W}^{\prime}(Q, P, F):=\mathcal{W}\left(P^{-1} Q^{T} F\right) .
$$

Then, the reversible stress, i.e., the first Piola-Kirchhoff stress (denoted henceforth by $\Pi)$ is given by $\partial W^{\prime} / \partial F(Q, P, F)$. We obtain

$$
\Pi=Q P^{-1} \mathbf{D} \mathcal{W}(E),
$$

so that, if $\rho_{0}$ denotes the mass density, $f$ the density of body forces (both in the undeformed configuration), while $\varphi$ denotes the transformation field, the equations of motion are

$$
\rho_{0} \frac{\partial^{2} \varphi}{\partial t^{2}}-\operatorname{div}\left[Q P^{-1} \mathbf{D} \mathcal{W}(E)\right]=f .
$$


The thermodynamic force ("back stress", denoted henceforth by $B$ ) associated with $P$ is given in turn by $-\partial W^{\prime} / \partial P(Q, P, F)$. We obtain

$$
B=-\frac{\partial W^{\prime}}{\partial P}(Q, P, F)=P^{-1} \mathbf{D} \mathcal{W}(E) E^{T} .
$$

The thermodynamic force ( "spin stress", denoted henceforth by $S$ ) associated with $Q$ is given by $-\partial W^{\prime} / \partial Q(Q, P, F)$. We obtain

$$
S=-Q P E[\mathbf{D} \mathcal{W}(E)]^{T} P^{-1} .
$$

The Cauchy stress $C$ (denoted henceforth by $C$ ) is given in terms of $\Pi$ through the (inverse Piola transform) as

We obtain

$$
C=\frac{1}{\operatorname{det} F} \Pi F^{T} .
$$

$$
C=\frac{1}{\operatorname{det} E} Q P^{-1} \mathbf{D} \mathcal{W}(E) E^{T} P Q^{T} .
$$

Because of frame indifference (see (2.1))

$$
\mathbf{D} \mathcal{W}(E)=E \mathbf{D} \hat{\mathcal{W}}^{s}\left(E^{T} E\right),
$$

where

$$
\mathbf{D} \hat{\mathcal{W}}^{s}(G):=\left(\mathbf{D} \hat{\mathcal{W}}(G)+\mathbf{D} \hat{\mathcal{W}}(G)^{T}\right)
$$

is the symmetrized derivative of $\hat{W}$. Then

$$
C=\frac{1}{\operatorname{det} E} Q P^{-1} E \mathbf{D} \hat{\mathcal{W}}^{s}\left(E^{T} E\right) E^{T} P Q^{T}=\frac{1}{\operatorname{det} E} Q P^{-1} A P Q^{T} ;
$$

in the relation above, the symmetric matrix $A$ is defined by

$$
A:=E \mathbf{D} \hat{\mathcal{W}}^{s}\left(E^{T} E\right) E^{T}\left(=\mathbf{D} \mathcal{W}(E) E^{T}\right) .
$$

Remark that, although $A$ is symmetric, $C$ is not automatically symmetric! But the symmetry of $C$ must be enforced because it is the direct consequence of the balance of moment of momentum.

Remark 3.1. This apparent lack of a priori symmetry of the Cauchy stress tensor is, to our knowledge, rather unusual. In the case of hyperelasticity that symmetry is a direct consequence of frame indifference because the Cauchy stress tensor then reduces to $A$ given in (3.6).

In any case, we should impose the symmetry of $P^{-1} A P$, or still

$$
A P^{2}=P^{2} A \text {. }
$$

Since $P^{2}$ is diagonal the above commutativity property is false unless $A$ is diagonal in the basis $\left\{\vec{e}_{i}\right\}$, or $P$ is the identity (since $\operatorname{det} P=1$ ). Thus, we conclude that the following hard constraint must be satisfied

$$
P=I \text { or } A=\mathbf{D} \mathcal{W}(E) E^{T} \text { is diagonal in the basis }\left\{\vec{e}_{i}\right\} .
$$

On the other hand, expressing $B, S$ in terms of $C$ yields

$$
\left\{\begin{array}{l}
B=\operatorname{det} E Q^{T} C Q P^{-1} \\
S=-\operatorname{det} E C^{T} Q .
\end{array}\right.
$$

In the absence of thermal dissipation, Clausius-Duhem's inequality reduces to

$$
B \cdot \dot{P}+S \cdot \dot{Q} \geq 0,
$$


or still, using (3.8),

$$
\operatorname{det} E Q^{T} C Q \cdot\left(\dot{P} P^{-1}-\dot{Q}^{T} Q\right) \geq 0 .
$$

(Note that the derivation of (3.10) has not used the symmetry of $C$ ).

Now, as already noted, $\operatorname{det} P \equiv 1$ throughout the evolution, so that, by time differentiation,

$$
\operatorname{tr} \dot{P} P^{-1}=0 .
$$

Similarly, since $Q \in S O(N), \dot{Q}^{T} Q+Q^{T} \dot{Q}=0$, so

$$
\dot{Q}^{T} Q \text { is skew-symmetric and } \operatorname{tr} \dot{Q}^{T} Q=0 .
$$

In view of $(3.11),(3.12),(3.10)$ reads as

$$
\operatorname{det} E Q^{T} C_{D} Q \cdot\left(\dot{P} P^{-1}-\dot{Q}^{T} Q\right) \geq 0 .
$$

We now appeal to the symmetry of $C$, hence of $C_{D}$. Because of (3.12) and since $\dot{P} P^{-1}=P^{-1} \dot{P},(3.13)$ becomes

$$
Q^{T} C_{D} Q \cdot \operatorname{det} E \dot{P} P^{-1} \geq 0 .
$$

In other words the variable $Q$ does not contribute to the dissipation!

Remark 3.2. Assuming for an instant that $W$ is isotropic, we remarked in Section 2 that we could then adopt the reverse decomposition $F=E P Q$ (the "classical" decomposition in the relevant literature) in lieu of (2.5). Then, proceeding as above, we would end up with

$$
\begin{aligned}
& \Pi=\mathbf{D} \mathcal{W}(E) P^{-1} Q \\
& B=E^{T} \mathbf{D} \mathcal{W}(E) P^{-1} \\
& S=-P^{-1} \mathbf{D} \mathcal{W}(E)^{T} E P Q \\
& C=\frac{1}{\operatorname{det} E} \mathbf{D} \mathcal{W}(E) E^{T}=\frac{1}{\operatorname{det} E} E \mathbf{D} \hat{\mathcal{W}}^{s}\left(E^{T} E\right) E^{T} .
\end{aligned}
$$

Consequently, the symmetry of the Cauchy stress tensor $C$ would be secured. However, the term $S \cdot \dot{Q}$ in the dissipation would then become

$$
-P^{-1} \mathbf{D} \mathcal{W}(E)^{T} E P Q \cdot \dot{Q}=-P^{-1} E^{T} \mathbf{D} \tilde{W}^{s}\left(E E^{T}\right) E P \cdot \dot{Q} Q^{T},
$$

with $W(E)=\tilde{W}\left(E E^{T}\right)$ where $\tilde{W}: \mathbb{M}_{s}^{N \times N} \rightarrow \overline{\mathbb{R}}$. Setting

$$
B:=E^{T} \mathbf{D} \tilde{W}^{s}\left(E E^{T}\right) E,
$$

we would conclude, in analogy with (3.7), that $B$ must be diagonal in the basis $\left\{\vec{e}_{i}\right\}$ so as to cancel the term $S \cdot \dot{Q}$ whenever $P \neq I$.

So, even in an isotropic setting, the $E P Q$-decomposition does not seem to alleviate the burden of a hard constraint of the type (3.7).

As stated in Section 2, a hallmark of plasticity should be the existence of a compact, convex set $\mathbf{K}$ containing 0 to which $C$, or more precisely here $C_{D}$, should belong. We thus impose that

$$
C_{D} \in \mathbf{K} \subset \mathbb{M}_{D}^{N \times N}
$$

That set should be frame indifferent, since $C$ is so. Hence $\mathbf{K}=Q^{T} \mathbf{K} Q, \forall Q \in$ $S O(N)$. 
The associated support function $\mathcal{H}$ defined as

$$
\mathcal{H}(T):=\sup \left\{C^{\prime} \cdot T: C^{\prime} \in \mathbf{K}\right\}
$$

will also be invariant under the action of $S O(N)$, that is

$$
\mathcal{H}\left(Q T Q^{T}\right)=\mathcal{H}(T), \forall Q \in S O(N) .
$$

Remark, for future use, that, in view of the convex, continuous and positively one-homogeneous character of $\mathcal{H}$, the following properties are easily checked:

$$
\begin{aligned}
& \partial \mathcal{H}(0)=\mathbf{K} \\
& \partial \mathcal{H}(M) \subset \mathbf{K}, M \in \mathbb{M}_{D}^{N \times N} .
\end{aligned}
$$

The normality rule (2.3) then reads as

$$
Q^{T} C_{D} Q \in \partial \mathcal{H}\left(\operatorname{det} E \dot{P} P^{-1}\right)
$$

(or, equivalently, $\operatorname{det} E \dot{P} P^{-1} \in N_{\mathbf{K}}\left(Q^{T} C_{D} Q\right)$ ). If we recall expression (3.5) for the Cauchy stress $C$ and (3.7) we thus finally get

or still

$$
\frac{1}{\operatorname{det} E} A_{D} \in \partial \mathcal{H}\left(\operatorname{det} E \dot{P} P^{-1}\right)
$$

$$
\frac{1}{\operatorname{det} E}\left[\mathbf{D} \mathcal{W}(E) E^{T}\right]_{D} \in \partial \mathcal{H}\left(\operatorname{det} E \dot{P} P^{-1}\right) .
$$

Summing up and recalling $(2.5),(3.2),(3.16),(3.17)$, the elasto-plastic evolution reads as

Model 3.3 (Elasto-Plastic Evolution). The transformation field $\varphi$ characterizing the evolution, for a given mass density $\rho_{0}$ and a given density of loads $f$ satisfies

$$
\nabla \varphi=\left\{\begin{array}{l}
Q P E \\
P \text { diagonal in a fixed orthonormal basis with positive eigenvalues } \\
\operatorname{det} P=1, \operatorname{det} E>0
\end{array}\right.
$$

$$
\begin{array}{r}
\rho_{0} \frac{\partial^{2} \varphi}{\partial t^{2}}-\operatorname{div}\left[Q P^{-1} \mathbf{D} \mathcal{W}(E)\right]=f \\
\frac{1}{\operatorname{det} E}\left[\mathbf{D} \mathcal{W}(E) E^{T}\right]_{D} \in \partial \mathcal{H}\left(\operatorname{det} E \dot{P} P^{-1}\right) \\
P=I \text { or } \mathbf{D} \mathcal{W}(E) E^{T}=E \mathbf{D} \hat{\mathcal{W}}^{s}\left(E^{T} E\right) E^{T} \text { is diagonal. }
\end{array}
$$

Of course, this system should be complemented with an appropriate set of initial and boundary conditions. At this point, we merely wish to draw attention to the role of the rotation $Q$ : it rotates the corresponding Piola-Kirchhoff stress so as to ensure that the equations of motion - the equilibrium equations in a quasi-static setting - are satisfied. Clearly, frame indifference is satisfied by the model because a pre-multiplication of the deformation gradient by a rotation $Q^{\prime}$ will result (see (3.5)) in changing the Cauchy stress $C$ into $Q^{\prime} C Q^{\prime T}$.

The next section will demonstrate that, upon assuming enough smoothness of all relevant fields, the evolution described in Model 3.3 can be rephrased - when in a quasi-static setting - as a variational evolution. 
Remark 3.4. The evolution obtained above is, as already mentioned, partially characterized by a set of admissible Cauchy stresses $\mathbf{K}$, at least through their deviatoric part. If instead, we a priori privilege a different measure of stress, to wit, the Kirchhoff stress $K$ defined as

$$
K=\operatorname{det} F C=\operatorname{det} E C,
$$

then a different elasto-plastic evolution is obtained. The difference lies in the disappearance of the term $\operatorname{det} E(t)$ from the flow rule (3.20). That equation thus becomes

$$
\left[\mathbf{D} \mathcal{W}(E) E^{T}\right]_{D} \in \partial \mathcal{H}\left(\dot{P} P^{-1}\right),
$$

all other equations remaining unchanged.

It will be seen later in Remark 4.5 that the resulting evolution can be equally rephrased, when in a quasi-static setting, as a variational evolution. That evolution will be subsequently analyzed in Section 5 because it proves mathematically more palatable than that generated by the Cauchy stress, precisely because of the absence of the determinant term; see the beginning of Section 5 .

Of course, this is not entirely satisfactory from our standpoint since Cauchy was part of our predicates in Section 2. We duly acknowledge this while pointing out that imposing a yield limit on the Kirchhoff stress is common occurrence in the literature on plasticity [?], [36, Section 18.2.8].

\section{Quasi-Static evolution - A variational evolution}

In this section, we specialize the variational evolution of Model 3.3 to the quasistatic case, which amounts to letting the term $\rho_{0} \partial^{2} \varphi / \partial t^{2}$ drop out of (3.19).

We propose to demonstrate that a blatant disregard of regularity issues allows one to reformulate that evolution within the framework advocated by A. MiELKE [33] for rate independent quasi-static evolutions. In a nutshell, such evolutions are characterized by a global minimality principle for the state variables at each time, together with an energy conservation statement. Such a formulation has been quite successful in handling a variety of rate independent evolutions ranging from fracture evolution to damage and small strain elasto-plasticity.

The global minimality principle alluded to in the previous paragraph is fully justified whenever the energy to be minimized is convex; such is the case in e.g. small strain elasto-plasticity (see [9]). However, in most settings convexity is lacking. Then the argument that restricts the variational evolution to global minimizers should be viewed as a convenient mathematical expedient to secure existence of such an evolution; such is the case in e.g. fracture (see $[12,17])$. In the absence of convexity, the equivalence between the original evolution system and the variational evolution is usually established upon replacing global minimality by an adequate notion of stationarity (see e.g. [4] still in the context of fracture).

In the current setting, convexity will be lacking so that stationarity will replace global minimality. However, for simplicity sake, we will illustrate the derivation as if the global minimality principle still held true.

Proposition 4.1 (Variational Evolution). In the absence of kinetic energy, the elasto-plastic evolution of Model 3.3, complemented by the boundary conditions

$$
\begin{array}{r}
\varphi=w(t) \text { on } \Gamma_{d} \subset \Omega, \text { with } w(t) \text { extended to all of } \mathbb{R}^{N} \\
\Pi(t) \nu=g(t) \text { on } \Gamma_{t}:=\partial \Omega \backslash \bar{\Gamma}_{d},
\end{array}
$$


(where $\Pi(t)$ is the first Piola-Kirchhoff stress defined in (3.1)) is satisfied by any solution of the following variational evolution:

Find $(\varphi(t), E(t), P(t), L(t), Q(t))$ with

- $P(t)$ diagonal in a fixed orthonormal basis $\left\{\vec{e}_{i}\right\}$ with positive eigenvalues, $\operatorname{det} P(t)=1 ; Q(t) \in S O(3)$;

- $\log P(t)=L(t)$

- $\nabla \varphi(t)=Q(t) P(t) E(t)$;

- $\varphi(t)=w(t)$ on $\Gamma_{d}$

and such that

(Global Minimality) For all $t \geq 0$ 's, the field $(E(t), P(t))$ is a global minimizer of

$$
\int_{\Omega} \mathcal{W}\left(E^{\prime}\right) d x-\int_{\Omega} f(t) \cdot \varphi^{\prime} d x-\int_{\Gamma_{t}} g(t) \cdot \varphi^{\prime} d \mathcal{H}^{N-1}+\int_{\Omega} \mathcal{H}\left(\operatorname{det} E(t)\left(L^{\prime}-L(t)\right)\right) d x
$$

among all $\left(\varphi^{\prime}, E^{\prime}, P^{\prime}, L^{\prime}, Q^{\prime}\right)$ with

$$
\left\{\begin{array}{l}
\nabla \varphi^{\prime}=Q^{\prime} P^{\prime} E^{\prime}, L^{\prime}=\log P^{\prime} \\
\varphi^{\prime} \equiv w(t) \text { on } \Gamma_{d} \\
P^{\prime} \text { diagonal in the basis }\left\{\vec{e}_{i}\right\} \text { with positive eigenvalues } \\
\operatorname{det} P^{\prime}=1, Q^{\prime} \in S O(3)
\end{array}\right.
$$

(Energy Conservation)

$$
\begin{gathered}
\frac{d}{d t}\left\{\int_{\Omega} \mathcal{W}\left(E(t) d x-\int_{\Omega} f(t) \cdot \varphi(t) d x-\int_{\Gamma_{t}} g(t) \cdot \varphi(t) d \mathcal{H}^{N-1}\right\}+\right. \\
\int_{\Omega} \mathcal{H}(\operatorname{det} E(t) \dot{L}(t)) d x=\int_{\Omega} Q(t) P^{-1}(t) \mathbf{D} \mathcal{W}(E(t)) \cdot \nabla \dot{w}(t) d x- \\
\int_{\Omega} \dot{f}(t) \cdot \varphi(t) d x-\int_{\Gamma_{t}} \dot{g}(t) \cdot \varphi(t) d \mathcal{H}^{N-1}-\int_{\Omega} f(t) \cdot \dot{w}(t) d x-\int_{\Gamma_{t}} g(t) \cdot \dot{w}(t) d \mathcal{H}^{N-1} ;
\end{gathered}
$$

Remark 4.2. Note that we do not impose the diagonality constraint (3.21). As will be seen in the proof below, this is a consequence of Global Minimality.

Remark 4.3. Because both $P(t)$ and the test plastic strains $P^{\prime}$ are isochoric (their determinants is identically 1), $L(t)$, as well as $L^{\prime}$ are in $\mathbb{M}_{D}^{N \times N}$, so that their presence in a term involving the dissipation potential $\mathcal{H}$ is meaningful.

Remark 4.4. In this formulation, a compatible initial condition for the elastoplastic evolution of Model 3.3 is one which satisfies the global minimality principle at time $t=0$.

Remark 4.5. In the context of Remark 3.4, the analogue of Proposition 4.1 holds at the expense of dropping the term $\operatorname{det} E(t)$ from both the Global Minimality and the Energy Conservation statements. 
The Energy Conservation of Proposition 4.1 can be integrated over the time interval $[0, t]$. It becomes

$$
\begin{gathered}
\int_{\Omega} \mathcal{W}\left(E(t) d x-\int_{\Omega} f(t) \cdot \varphi(t) d x-\int_{\Gamma_{t}} g(t) \cdot \varphi(t) d \mathcal{H}^{N-1}+\int_{0}^{t} \int_{\Omega} \mathcal{H}(\operatorname{det} E(s) \dot{L}(s)) d x d s\right. \\
=\int_{0}^{t} \int_{\Omega} Q(s) P^{-1}(s) \mathbf{D} \mathcal{W}(E(s)) \cdot \nabla \dot{w}(s) d x d s-\int_{0}^{t} \int_{\Omega} \dot{f}(s) \cdot \varphi(s) d x d s- \\
\int_{0}^{t} \int_{\Gamma_{t}} \dot{g}(s) \cdot \varphi(s) d \mathcal{H}^{N-1} d s-\int_{0}^{t} \int_{\Omega} f(s) \cdot \dot{w}(s) d x d s-\int_{0}^{t} \int_{\Gamma_{t}} g(s) \cdot \dot{w}(s) d \mathcal{H}^{N-1} d s
\end{gathered}
$$

The term $\int_{0}^{t} \int_{\Omega} \mathcal{H}(\operatorname{det} E(s) \dot{L}(s)) d x d s$ in the expression above presupposes some type of regularity in time of $L(t)=\log P(t)$.

Define, for $t_{1}, t_{2} \in[0, T]$, with $t_{1} \leq t_{2}$, the total dissipation as

$\operatorname{Diss}_{\mathcal{H}}\left(t_{1}, t_{2}\right):=$

$$
\begin{array}{r}
\lim _{\delta \searrow 0}\left\{\sum_{i=1}^{N_{\delta}} \int_{\Omega} \operatorname{det} E\left(s_{i}^{\delta}\right) \mathcal{H}\left(L\left(s_{i}^{\delta}\right)-L\left(s_{i-1}^{\delta}\right)\right) d x:\left\{s_{i}^{\delta}\right\} \text { finite partitions of }\left[t_{1}, t_{2}\right] ;\right. \\
\left.\sup \left\{s_{i}^{\delta}-s_{i-1}^{\delta}\right\} \leq \delta\right\} .
\end{array}
$$

Then, using the positive one-homogeneity of $\mathcal{H}$, the term $\int_{0}^{t} \int_{\Omega} \mathcal{H}(\operatorname{det} E(s) \dot{L}(s)) d x d s$ in the Energy Conservation can be unambiguously replaced by $\operatorname{Diss} \mathcal{H}(0, t)$, provided that all involved fields are sufficiently smooth, as demonstrated in [10, Lemma 8.2]; the value of $\operatorname{Diss} \mathcal{H}\left(t_{1}, t_{2}\right)$ does not depend upon the particular sequence of partitions $\left\{s_{i}^{\delta}\right\}$.

We can restate the variational evolution in Proposition 4.1 as a definition which, if satisfied by a quintuplet $(\varphi(t), E(t), P(t), L(t), Q(t))$ will ensure satisfaction of the elasto-plastic evolution of Model 3.3 of in the absence of kinetic energy and for the boundary conditions (4.1).

Definition 4.6. A variational evolution $(\varphi(t), E(t), P(t), L(t), Q(t))$ is defined as

- $P(t)$ diagonal in a fixed orthonormal basis $\left\{\vec{e}_{i}\right\}$ with positive eigenvalues, $\operatorname{det} P(t)=1 ; Q(t) \in S O(3)$;

- $\log P(t)=L(t)$

- $\nabla \varphi(t)=Q(t) P(t) E(t)$

- $\varphi(t)=w(t)$ on $\Gamma_{d}$

and is such that

(Global Minimality) For all $t \geq 0$ 's, the field $(E(t), P(t))$ is a global minimizer of

$$
\int_{\Omega} \mathcal{W}\left(E^{\prime}\right) d x-\int_{\Omega} f(t) \cdot \varphi^{\prime} d x-\int_{\Gamma_{t}} g(t) \cdot \varphi^{\prime} d \mathcal{H}^{N-1}+\int_{\Omega} \mathcal{H}\left(\operatorname{det} E(t)\left(L^{\prime}-L(t)\right)\right) d x,
$$

among all $\left(\varphi^{\prime}, E^{\prime}, P^{\prime}, L^{\prime}, Q^{\prime}\right)$ with

$$
\left\{\begin{array}{l}
\nabla \varphi^{\prime}=Q^{\prime} P^{\prime} E^{\prime}, L^{\prime}=\log P^{\prime} \\
\varphi^{\prime} \equiv w(t) \text { on } \Gamma_{d} \\
P^{\prime} \text { diagonal in the basis in the basis }\left\{\vec{e}_{i}\right\} \text { with positive eigenvalues } \\
\operatorname{det} P^{\prime}=1, Q^{\prime} \in S O(3)
\end{array}\right.
$$


(Energy Conservation) ${ }^{\star}$

$$
\begin{gathered}
\int_{\Omega} \mathcal{W}\left(E(t) d x-\int_{\Omega} f(t) \cdot \varphi(t) d x-\int_{\Gamma_{t}} g(t) \cdot \varphi(t) d \mathcal{H}^{N-1}+\operatorname{Diss} \mathcal{H}(0, t)=\right. \\
\int_{0}^{t} \int_{\Omega} Q(s) P^{-1}(s) \mathbf{D} \mathcal{W}(E(s)) \cdot \nabla \dot{w}(s) d x d s-\int_{0}^{t} \int_{\Omega} \dot{f}(s) \cdot \varphi(s) d x d s \\
-\int_{0}^{t} \int_{\Gamma_{t}} \dot{g}(s) \cdot \varphi(s) d \mathcal{H}^{N-1} d s-\int_{0}^{t} \int_{\Omega} f(s) \cdot \dot{w}(s) d x d s-\int_{0}^{t} \int_{\Gamma_{t}} g(s) \cdot \dot{w}(s) d \mathcal{H}^{N-1} d s .
\end{gathered}
$$

Remark 4.7. Once again, a definition of a variational evolution can be readily derived in the context of Remarks 3.4, 4.5 at the expense of dropping $\operatorname{det} E(t)$ from the Global minimality statement, and also of redefining Diss $\mathcal{H}$ as

$$
\operatorname{Diss}_{\mathcal{H}}\left(t_{1}, t_{2}\right):=\sup _{\left\{s_{i}\right\} \text { finite partitions of }\left[t_{1}, t_{2}\right]}\left\{\sum_{i=1}^{N_{\delta}} \int_{\Omega} \mathcal{H}\left(L\left(s_{i}\right)-L\left(s_{i-1}\right)\right) d x\right\},
$$

which is a more classical definition of the dissipation.

In all fairness, the legitimacy of the substitution of the dissipation term in the energy conservation is doubtful at present because the required smoothness of the fields $E(t), L(t)$ is lacking, even in the regularized context of Section 5 below.

Proof of Proposition 4.1. Because $P(t), P^{\prime}$ are diagonal with only positive eigenvalues, one can trivially define their logarithms $L(t), L^{\prime}$.

Then, formally

$$
\dot{P}(t) P^{-1}(t)=P^{-1}(t) \dot{P}(t)=\dot{L}(t) .
$$

Step 1- Consequences of global minimality: Assume global minimality and choose $\varphi^{\prime}=\varphi(t), E^{\prime}=\bar{P}^{-1} E(t), P^{\prime}=P(t) \bar{P}, Q^{\prime}=Q(t)$. Then,

$$
\int_{\Omega} \mathcal{W}(E(t)) d x \leq \int_{\Omega} \mathcal{W}\left(\bar{P}^{-1} E(t)\right) d x+\int_{\Omega} \mathcal{H}(\operatorname{det} E(t) \log \bar{P}) d x .
$$

Take $\bar{P}=Q " \exp \left(\chi_{B} \varepsilon M\right) Q " T$ with $M \in \mathbb{M}_{D}^{N \times N}, B$ an arbitrary Borel set and $Q " \in S O(N)$ such that $Q " M Q " T$ is diagonal in the same basis as $P(t)$. Then,

$$
\log \bar{P}=\varepsilon \chi_{B} Q " M Q " T+o(\varepsilon) .
$$

From (4.3), we obtain

$0 \leq \int_{B} \mathbf{D} \mathcal{W}(E(t)) \cdot[Q " \exp (-\varepsilon M) Q " T-I] E(t)+o(\varepsilon)+\varepsilon \int_{B} \mathcal{H}(\operatorname{det} E(t) Q " M Q " T)$,

that is,

$$
0 \leq \int_{B} \mathbf{D} \mathcal{W}(E(t)) \cdot Q "[-\varepsilon M] Q " T E(t)+o(\varepsilon)+\varepsilon \int_{B} \mathcal{H}\left(\operatorname{det} E(t) Q " M Q{ }^{T}\right) .
$$

Choosing $N:=\operatorname{det} E(t) Q " M Q " T$, dividing by $\varepsilon>0$ and letting $\varepsilon$ tend to 0 yields

$$
\int_{B} \frac{1}{\operatorname{det} E(t)} \mathbf{D} \mathcal{W}(E(t)) E(t)^{T} \cdot N \leq \int_{B} \mathcal{H}(N)
$$

which is equivalent to

$$
\frac{1}{\operatorname{det} E(t)}\left[\mathbf{D} \mathcal{W}\left(E(t) E^{T}(t)\right]_{D} \in \partial \mathcal{H}(0)=\mathbf{K}\right.
$$


Remark that we cannot obtain the equality in (4.4) because we are not allowed to choose $\varepsilon<0$ since $\mathcal{H}$ is only positively one-homogeneous.

Choose now

$$
\varphi^{\prime}=\varphi(t)+\varepsilon \psi, \psi \in C_{c}^{\infty}\left(\Omega \cup \Gamma_{t}\right),
$$

and consider the decomposition

$$
\nabla \varphi^{\prime}=Q(t) P(t)\left[E(t)+\varepsilon P^{-1}(t) Q^{T}(t) \nabla \psi\right] .
$$

We are at liberty to take

$$
E^{\prime}=E(t)+\varepsilon P^{-1}(t) Q^{T}(t) \nabla \psi
$$

without changing $P(t)$. Inserting the test pair $\left(E^{\prime}(t), P(t)\right)$ into global minimality yields

$$
\begin{aligned}
& \int_{\Omega} \mathcal{W}(E(t)) d x \leq \\
& \int_{\Omega} \mathcal{W}\left(E(t)+\varepsilon P^{-1}(t) Q^{T}(t) \nabla \psi\right) d x-\varepsilon\left\{\int_{\Omega} f(t) \cdot \psi d x-\int_{\Gamma_{t}} g(t) \cdot \psi d \mathcal{H}^{N-1}\right\},
\end{aligned}
$$

that is

$$
\begin{array}{r}
-\varepsilon\left\{\int_{\Omega} \mathbf{D} \mathcal{W}(E(t)) \cdot P^{-1}(t) Q^{T}(t) \nabla \psi+\int_{\Omega} f(t) \cdot \psi d x-\int_{\Gamma_{t}} g(t) \cdot \psi d \mathcal{H}^{N-1}\right\} \\
+o(\varepsilon) \leq 0,
\end{array}
$$

or still

$$
\int_{\Omega} Q(t) P^{-1}(t) \mathbf{D} \mathcal{W}(E(t)) \cdot \nabla \psi-\int_{\Omega} f(t) \cdot \psi d x-\int_{\Gamma_{t}} g(t) \cdot \psi d \mathcal{H}^{N-1}=0 .
$$

Choosing $\psi \in C_{c}^{\infty}(\Omega)$ immediately yields the equilibrium equation (3.19) (without the acceleration term), and then choosing $\psi \in C_{c}^{\infty}\left(\Omega \cup \Gamma_{t}\right)$ and recalling (3.1) yields the Neumann boundary condition

$$
\Pi(t) \nu=g(t) \text { on } \Gamma_{t} .
$$

Finally, take $\zeta$ to be an arbitrary element of $C_{c}^{\infty}(\Omega)$ and $B$ to be an arbitrary skew-symmetric matrix and note that $\exp \varepsilon \zeta B \in S O(N)$ for $\epsilon$ small enough. Choose $\varphi^{\prime}=\varphi(t), E^{\prime}=P^{-1}(t) \exp (-\varepsilon \zeta B) P(t) E(t), P^{\prime}=P(t), Q^{\prime}=Q(t) \exp (\varepsilon \zeta B)$. Then, inserting the test pair $\left(E^{\prime}(t), P(t)\right)$ into global minimality yields

$$
\int_{\Omega} \mathcal{W}(E(t)) d x \leq \int_{\Omega} \mathcal{W}\left(P^{-1}(t) \exp (-\varepsilon \zeta B) P(t) E(t)\right) d x
$$

that is

$$
\varepsilon \int_{\Omega}\left(P^{-1}(t) \mathbf{D} \mathcal{W}(E(t)) E^{T}(t) P(t) \cdot B\right) \zeta d x-o(\varepsilon) \leq 0,
$$

or still, upon localizing,

$$
P^{-1}(t) \mathbf{D} \mathcal{W}(E(t)) E^{T}(t) P(t) \cdot B=0, \forall B \text { skew-symmetric. }
$$

Relation (4.7) implies that $P^{-1}(t) \mathbf{D} \mathcal{W}(E(t)) E^{T}(t) P(t)=P^{-1}(t) A(t) P(t)$ (see (3.6)) is symmetric, hence, reproducing the argument leading to (3.7), that (3.21) holds true. 
Step 2- Consequences of energy conservation: Using (3.19), (4.6), the following string of equalities holds true:

$$
\begin{gathered}
\int_{\Omega} \mathbf{D} \mathcal{W}(E(t)) \cdot \dot{E}(t) d x+\int_{\Omega} \mathcal{H}(\operatorname{det} E(t) \dot{L}(t)) d x= \\
\int_{\Omega} Q(t) P^{-1}(t) \mathbf{D} \mathcal{W}(E(t)) \cdot Q(t) P(t) \dot{E}(t) d x+\int_{\Omega} \mathcal{H}(\operatorname{det} E(t) \dot{L}(t)) d x= \\
\int_{\Omega} Q(t) P^{-1}(t) \mathbf{D} \mathcal{W}(E(t)) \cdot \nabla \dot{\varphi}(t) d x-\int_{\Omega} Q(t) P^{-1}(t) \mathbf{D} \mathcal{W}(E(t)) . \\
(\dot{Q}(t) P(t)+Q(t) \dot{P}(t)) E(t) d x+\int_{\Omega} \mathcal{H}(\operatorname{det} E(t) \dot{L}(t)) d x= \\
\int_{\Omega} Q(t) P^{-1}(t) \mathbf{D} \mathcal{W}(E(t)) \cdot \nabla \dot{w}(t) d x+\int_{\Gamma_{t}} g(t) \cdot(\dot{\varphi}(t)-\dot{w}(t)) d \mathcal{H}^{N-1}+ \\
\int_{\Omega} f(t) \cdot(\dot{\varphi}(t)-\dot{w}(t)) d x-\int_{\Omega} Q(t) P^{-1}(t) \mathbf{D} \mathcal{W}(E(t)) \cdot(\dot{Q}(t) P(t)+Q(t) \dot{P}(t)) E(t) d x \\
+\int_{\Omega} \mathcal{H}(\operatorname{det} E(t) \dot{L}(t)) d x .
\end{gathered}
$$

But, according to energy conservation,

$$
\begin{aligned}
& \int_{\Omega} \mathbf{D} \mathcal{W}(E(t)) \cdot \dot{E}(t) d x+\int_{\Omega} \mathcal{H}(\operatorname{det} E(t) \dot{L}(t)) d x \\
& \qquad \begin{aligned}
\int_{\Omega} Q(t) P^{-1}(t) \mathbf{D} \mathcal{W}(E(t)) \cdot \nabla \dot{w}(t) d x & +\int_{\Omega} f(t) \cdot(\dot{\varphi}(t)-\dot{w}(t)) d x \\
& +\int_{\Gamma_{t}} g(t) \cdot\left(\dot{\varphi}(t)-\dot{w}(t) d \mathcal{H}^{N-1} .\right.
\end{aligned}
\end{aligned}
$$

Comparing the two expressions above, we conclude that

$$
\begin{array}{r}
\int_{\Omega} Q(t) P^{-1}(t) \mathbf{D} \mathcal{W}(E(t)) \cdot(\dot{Q}(t) P(t)+Q(t) \dot{P}(t)) E(t) d x \\
=\int_{\Omega} \mathcal{H}(\operatorname{det} E(t) \dot{L}(t)) d x
\end{array}
$$

Recalling definition (3.6),

$$
Q(t) P^{-1}(t) \mathbf{D} \mathcal{W}(E(t)) \cdot \dot{Q}(t) P(t) E(t)=P^{-1}(t) A(t) P(t) \cdot Q^{T}(t) \dot{Q}(t) .
$$

But, according to the already proved diagonality property $(3.21), A(t)$ commutes with $P(t)$, so that the relation above becomes

$$
Q(t) P^{-1}(t) \mathbf{D} \mathcal{W}(E(t)) \cdot \dot{Q}(t) P(t) E(t)=A(t) \cdot Q^{T}(t) \dot{Q}(t)
$$

and, since the left hand-side of the dot product is symmetric while the right handside is skew-symmetric, that expression is identically null. Thus, (4.8) becomes

$$
\int_{\Omega} Q(t) P^{-1}(t) \mathbf{D} \mathcal{W}(E(t)) \cdot Q(t) \dot{P}(t) E(t) d x=\int_{\Omega} \mathcal{H}(\operatorname{det} E(t) \dot{L}(t)) d x,
$$

or still, since tr $P^{-1}(t) \dot{P}(t)=0$,

$$
\int_{\Omega}\left[\mathbf{D} \mathcal{W}(E(t)) E^{T}(t)\right]_{D} \cdot P^{-1}(t) \dot{P}(t) d x=\int_{\Omega} \mathcal{H}(\operatorname{det} E(t) \dot{L}(t)) d x .
$$


Relation (4.5) implies in particular that

$$
\frac{1}{\operatorname{det} E(t)}\left[\mathbf{D} \mathcal{W}(E(t)) E^{T}(t)\right]_{D} \cdot \operatorname{det} E(t) \dot{L}(t) \leq \mathcal{H}(\operatorname{det} E(t) \dot{L}(t)) .
$$

Since $\dot{L}(t)=\dot{P}(t) P^{-1}(t)=P^{-1}(t) \dot{P}(t)$, this also reads as

$$
\frac{1}{\operatorname{det} E(t)}\left[\mathbf{D} \mathcal{W}(E(t)) E^{T}(t)\right]_{D} \cdot \operatorname{det} E(t) \dot{P}(t) P^{-1}(t) \leq \mathcal{H}(\operatorname{det} E(t) \dot{L}(t)) .
$$

Thus, in view of (4.9), inequality (4.10) is an equality, that is

$$
\left.\frac{1}{\operatorname{det} E(t)}\left[\mathbf{D} \mathcal{W}(E(t)) E^{T}(t)\right]_{D} \cdot \operatorname{det} E(t) \dot{P}(t) P^{-1}(t)\right)=\mathcal{H}(\operatorname{det} E(t) \dot{L}(t)) .
$$

Convex duality then permits to conclude, in view of (4.5), that (3.20) is satisfied.

Remark 4.8. As already mentioned at the onset of this section, the variational evolution (if it exists) has been shown to satisfy (in the absence of kinetic energy) the elasto-plastic evolution of Model (3.3) under the assumption of global minimality. In lieu of the latter property, a proper notion of stationarity would yield a similar result, although we do not wish to elaborate further on that notion in this work.

Remark 4.9 (Mielke's model). To our knowledge, A. Mielke [30] was the first to establish that finite elasto-plasticity can be reformulated - in a quasi-static setting - as a variational evolution. In this remark we propose to contrast our approach with that which he subsequently developed, together with his collaborators.

In his formulation (see e.g. [31, 32]), the adopted multiplicative decomposition is $F=E P^{-1}$, and not $F=P E$ as in the present work. (The consideration of $P^{-1}$ in lieu of $P$ is purely for notational convenience.)

In that setting, it is easily seen that the Cauchy stress tensor is given by

$$
C=\frac{1}{\operatorname{det} E} \mathbf{D} \mathcal{W}(E) E^{T},
$$

while the back stress is given by

$$
B=-P^{-T} E^{T} \mathbf{D} \mathcal{W}(E) .
$$

The author chooses to constrain the tensor $P^{T} B=E^{T} \mathbf{D} \mathcal{W}(E)$ to belong to some convex set $\mathbf{K}$, so that his flow rule is of the form

$$
P^{-1} \dot{P} \in N_{\mathbf{K}}\left(E^{T} \mathbf{D} \mathcal{W}(E)\right),
$$

or equivalently,

$$
E^{T} \mathbf{D} \mathcal{W}(E) \in \mathcal{H}\left(P^{-1} \dot{P}\right)
$$

with notation that should be clear to the reader.

The choice of the decomposition $F=E P^{-1}$ leaves no choice as to the stress that should be constrained. In particular, it does not seem possible to marry the decomposition with a constraint on the Cauchy stress.

Further, the lack of commutativity between $P^{-1}$ and $\dot{P}$ prevents the use of a variational formulation for the resulting evolution. The author has to modify the dissipation so as to introduce a dissipation functional with the required subadditivity and homogeneity properties. Specifically, he introduces the dissipation 
potential -which he calls the induced dissipation distance - as

$\mathcal{D}\left(P, P^{\prime}\right):=\inf \left\{\int_{0}^{1} \mathcal{H}\left(P^{-1}(s) \dot{P}(s)\right) d s: P\right.$ smooth on $\left.[0,1] ; P(0)=P, P(1)=P^{\prime}\right\}$,

and it is for that new dissipation potential that he proposes a variational evolution. See [30, Section 2]. In that formulation the total energy dissipated over the time interval $[0, t]$ - that is $\int_{0}^{t} \int_{\Omega} \mathcal{H}(\operatorname{det} E(s) \dot{L}(s)) d x d s$ if integrating in time the energy conservation in Proposition 4.1 - has to be replaced by a total variation type expression, namely

$$
\sup \left\{\sum_{i=0, \ldots, p} \int_{\Omega} \mathcal{D}\left(P\left(t_{i-1}\right), P\left(t_{i}\right)\right) d x:\left\{t_{i}\right\} \text { with } t_{0}=0 \leq t_{1} \ldots \leq t_{p}=t\right\}
$$

A solution to such a variational evolution may not satisfy the corresponding elasto-plastic evolution precisely because of this dissipation gap introduced through the infimum in the definition of $\mathcal{D}$.

In view of the tremendous successes of variational evolutions for a wide variety of rate independent evolutions (at least when global minimality is enforced), it would be tempting to attempt a proof of the existence of evolution satisfying the criteria put forth in Proposition 4.1 (or, alternatively, in Remark 3.4). Unfortunately, this is a forbidding task on two grounds. On the one hand the energy to be minimized is the sum of two non-quadratic energies which would be a challenge even in the context of small strain elasto-plasticity. On the other hand, the dissipation is not convex because of the presence of the logarithmic term; further it has superlinear growth at 0 and sub-linear growth at $\infty$. Consequently, even the functional framework - that is that which ensures bounded energies and compactness of the minimizing sequences - is unclear to us at this point.

The model advocated in e.g. [30, 31, 32] was later regularized in [26] through the introduction of both a plasticity term and a gradient plasticity term in the energy that, together, enforce compactness of the plastic strains associated with the minimizing sequences. Schematically, in the simplest setting, the free energy becomes $\mathcal{W}(E)+\mathcal{W}_{\text {hard }}(P)+|\nabla P|^{r}, r>1$. The energy $\mathcal{W}_{\text {hard }}$ is often referred to as a hardening energy while the plastic gradient term is called just that, a plastic gradient. An existence theorem can then be proved under adequate assumptions on the energy density $\mathcal{W}$ and on the induced dissipation distance $\mathcal{D}$ of Remark 4.9; see [26, Theorem 3.1]. In the next section we quickly show that adding regularization terms which enforce compactness of the plastic strains and of the rotations produces a similar result, at least in the setting of Remarks 3.4, 4.5, 4.7.

\section{A Regularized Variational Evolution}

As previously announced, this section is devoted to a regularized model for which Sobolev-compactness of the plastic strains is immediate. For simplicity sake, we specialize the dimension to be $N=3$, but the results below could easily be adapted to any dimension.

Unfortunately the model for which existence will be proved also violates one of the basic requirements introduced in Section 2, namely that the yield threshold be formulated in terms of the Cauchy stress. In what follows that threshold is formulated in terms of the Kirchhoff stress as in Remark 3.4. We do so because 
the presence of the term $\operatorname{det} E(t)$ in the dissipation makes the analysis intractable. The energy conservation statement in Definition 4.6 seems unattainable for lack of any kind of time estimate on $\log P$.

We list the constitutive assumptions under which existence of a variational evolution can be ascertained. They should be thought of as similar to the assumptions in [20, Section 5] and of those in [29, Section 2], themselves inspired from [2, Section 2.4]. Our goal in this section is illustrative, so that, rather than strive for the utmost generality, we put forth the simplest constitutive assumptions, most notably on growth, or coercivity that will secure the desired result.

The body under consideration occupies a bounded Lipschitz domain $\Omega \subset \mathbb{R}^{3}$. We take $p$ with

$$
3<p<\infty,
$$

so that $W^{1, p}(\Omega)$ is compactly imbedded into $C^{0}(\bar{\Omega})$.

The elastic energy: $\mathcal{W}: \mathbb{M}^{3 \times 3} \rightarrow[0, \infty]$ is polyconvex and satisfies, for all $F \in \mathbb{M}_{+}^{3 \times 3}$,

H1. $\mathcal{W} \in C^{1}\left(\mathbb{M}_{+}^{3 \times 3}\right), \quad \mathcal{W} \equiv \infty$ on $\mathbb{M}^{3 \times 3} \backslash \mathbb{M}_{+}^{3 \times 3}$,

H2. $\mathcal{W}(I d)=0$;

H3. $\mathcal{W}(R F)=\mathcal{W}(F) \quad \forall R \in S O(3)$;

H4. $\mathcal{W}(F) \geq c_{1} \operatorname{dist}^{p}(F ; S O(3))$;

H5. $\left|\mathbf{D} \mathcal{W}(F) F^{T}\right| \leq c_{2}(\mathcal{W}(F)+1)$;

H6. There exists a modulus of continuity $\omega$ such that, for all $N \in \mathbb{M}_{+}^{3 \times 3}$,

$$
\left|\mathbf{D} \mathcal{W}(F) F^{T}-\mathbf{D} \mathcal{W}(N F)(N F)^{T}\right| \leq \omega(\|N-I d\|)(\mathcal{W}(F)+1) .
$$

Here $c_{1}, c_{2}$ are positive constants.

Remark 5.1. There are indeed classical energy densities, like those asociated with Ogden materials, that satisfy $\mathrm{H} 1-\mathrm{H} 6$, as demonstrated in [20, Remarks 5.1, 5.3].

The hardening functional: Exactly as in [29], we consider a severely constrained model of kinematic hardening (kinematic, so as not to introduce yet an additional internal variable). The hardening functional $\mathcal{W}_{\text {hard }}: \mathbb{R}_{+}^{3} \rightarrow[0, \infty]$ is of the form

$$
\mathcal{W}_{\text {hard }}(P):= \begin{cases}\widetilde{\mathcal{W}}_{\text {hard }}(P) & \text { for every } P \in \mathbf{V} \\ +\infty & \text { otherwise }\end{cases}
$$

The constraint is that $\mathbf{V}$ should be a compact subset of $\mathbb{R}_{1}^{3}$ that contains $1_{3}$ as an interior point. We also take the map $\widetilde{\mathcal{W}}_{\text {hard }}: \mathbb{R}_{1}^{3} \rightarrow[0, \infty)$ to be continuous.

In particular the hypotheses on $\mathbf{V}$ imply the existence of a constant $c$ such that

$$
\begin{aligned}
& |P|+\left|P^{-1}\right| \leq c \quad \text { for every } P \in \mathbf{V}, \\
& \left|P-1_{3}\right| \geq \frac{1}{c} \quad \text { for every } P \in \mathbb{R}_{1}^{3} \backslash \mathbf{V} .
\end{aligned}
$$

The dissipation functional : $\mathcal{H}: \mathbb{M}_{D}^{N \times N} \rightarrow[0,+\infty)$ is a convex, positively onehomogeneous function such that, for some $0<r<R$ and any $F \in \mathbb{M}_{D}^{N \times N}$,

$$
r|F| \leq \mathcal{H}(F) \leq R|F| .
$$

We also define, for any $L:[0, T] \rightarrow L^{1}\left(\Omega ; \mathbb{R}^{3}\right)$ and $0 \leq t_{1} \leq t_{2} \leq T$,

$$
\operatorname{Var}\left(t_{1}, t_{2} ; L\right):=\sup _{\left\{s_{i}\right\}}\left\{\sum_{i=1}^{N} \int_{\Omega}\left|L\left(s_{i}\right)-L\left(s_{i-1}\right)\right| d x: s_{0}=t_{1}<s_{1}<\ldots<s_{N}=t_{2}\right\} .
$$


The boundary conditions : On an open subset $\Gamma$ of $\partial \Omega$, we impose a Dirichlet boundary condition $\mathbf{g}:[0, T] \times \mathbb{R}^{3} \rightarrow \mathbb{R}^{3}$ where $\mathbf{g}(t, \cdot): \mathbb{R}^{3} \rightarrow \mathbb{R}^{3}$ is a global diffeomorphism satisfying

$$
\begin{aligned}
& \mathbf{g} \in C^{2}\left([0, T] \times \mathbb{R}^{3} ; \mathbb{R}^{3}\right), \\
& \|\nabla \mathbf{g}\|_{L^{\infty}} \leq C,\|\nabla \mathbf{g}\|_{L^{\infty}} \leq C,\|\nabla \ddot{\mathbf{g}}\|_{L^{\infty}} \leq C,\left\|(\nabla \mathbf{g})^{-1}\right\|_{L^{\infty}} \leq C .
\end{aligned}
$$

Remark 5.2. Note that, for simplicity sake, $\mathbf{g}$ is the only "loading process" for the system. Body or surface forces could be readily incorporated provided that they are endowed with adequate regularity.

Also, we have made no attempt to optimize the regularity assumptions on $\mathbf{g}$.

The deformations: The deformations $\varphi:[0, T] \times \Omega \rightarrow \mathbb{R}^{3}$ can then be decomposed as follows:

with

$$
\varphi(t, x)=\mathbf{g}(t, y(t, x))
$$

$$
y \in \mathscr{Y}:=\left\{y \in W^{1, p}\left(\Omega ; \mathbb{R}^{3}\right): y\left\lfloor_{\Gamma}=i d\right\} .\right.
$$

Hence in particular (see e.g. [34, Remark 2.2 and Proposition 2.2]),

$$
\nabla \varphi(t, x)=\nabla \mathbf{g}(t, y(t, x)) \nabla y(t, x)
$$

We define

$$
\mathscr{A}:=\left\{(y, Q, P) \in \mathscr{Y} \times W^{1, p}(\Omega ; S O(3)) \times W^{1, p}\left(\Omega ; \mathbb{R}_{1}^{3}\right)\right\} .
$$

The energy functional : The elastic and free energies at time $t$ are defined to be, for any $(y, Q, P) \in \mathscr{A}$,

$$
\left\{\begin{array}{l}
\mathcal{E}(t, y, Q, P):=\int_{\Omega} \mathcal{W}\left(P^{-1} Q^{T} \nabla \mathbf{g}(t, y) \nabla y\right) d x \\
\mathcal{F}(t, y, Q, P):=\mathcal{E}(t, y, Q, P)+\int_{\Omega} \mathcal{W}_{\text {hard }}(P) d x+\int_{\Omega}|\nabla P|^{p} d x+\int_{\Omega}|\nabla Q|^{p} d x .
\end{array}\right.
$$

Remark 5.3. The reader will note the presence of two gradient terms in the expression for the energy, one for $P$ and one for $Q$. This is to be expected, if recalling the setting of [26], because our decomposition of the deformation gradient involves both $P$ and $Q$, as already discussed earlier.

We establish a few properties of $\mathcal{E}$, hence of $\mathcal{F}$. First, under assumptions $\mathrm{H} 1, \mathrm{H} 5$, a straightforward adaptation of [20, Lemma 5.5] would demonstrate that, for any $(y, Q, P) \in \mathscr{A}$,

$$
t \rightarrow \mathcal{E}(t, y, Q, P)(\text { hence } \mathcal{F}(t, y, Q, P)) \text { is differentiable with derivative }
$$

$\partial_{t} \mathcal{E}\left(\right.$ resp. $\left.\partial_{t} \mathcal{F}\right)(t, y, Q, P)=\int_{\Omega} \mathbf{D} \mathcal{W}\left(P^{-1} Q^{T} \nabla \mathbf{g}(t, y) \nabla y\right) \cdot P^{-1} Q^{T} \nabla \dot{\mathbf{g}}(t, y) \nabla y d x$.

Next, the maps

$$
(t, y, Q, P) \rightarrow\left\{\begin{array}{l}
\mathcal{E}(t, y, Q, P) \\
\mathcal{F}(t, y, Q, P)
\end{array} \text { are weakly lower semi-continuous in }[0, T] \times \mathscr{A}\right.
$$

Indeed, if $\left(t_{k}, y_{k}, Q_{k}, P_{k}\right) \rightarrow(t, y, Q, P)$, weakly in $[0, T] \times \mathscr{A}$, then, since $p>3$, Rellich's theorem implies uniform convergence of that sequence in $[0, T] \times C^{0}\left(\bar{\Omega} ; \mathbb{R}^{3} \times\right.$ $\left.S O(3) \times \mathbb{R}_{1}^{3}\right)$. But, in view of $(5.4), \varphi_{k}:=g\left(t_{k}, y_{k}\right)$ also converges uniformly to 
$\varphi:=g(t, y)$ in $C^{0}\left(\bar{\Omega} ; \mathbb{R}^{3}\right)$ while, thanks to $(5.5)$ and (5.6) it also converges weakly to $\varphi$ in $W^{1, p}\left(\Omega ; \mathbb{R}^{3}\right)$.

Then, exactly as in the argument leading to (5.33) below (which we prefer to detail at that time),

$$
\int_{\Omega} \mathcal{W}\left(P^{-1} Q^{T} \nabla \varphi\right) d x \leq \liminf _{k} \int_{\Omega} \mathcal{W}\left(P_{k}^{-1} Q_{k}^{T} \nabla \varphi_{k}\right) d x
$$

so that

$$
\begin{aligned}
\liminf _{k} \mathcal{E}\left(t_{k}, y_{k}, Q_{k}, P_{k}\right)=\liminf _{k} \int_{\Omega} \mathcal{W}\left(P_{k}^{-1} Q_{k}^{T} \nabla \varphi_{k}\right) d x \\
\quad \geq \int_{\Omega} \mathcal{W}\left(P^{-1} Q^{T} \nabla \varphi\right) d x=\mathcal{E}(t, y, Q, P) .
\end{aligned}
$$

The weak lower semi-continuity of $\mathcal{F}$ is then established as in (5.24), (5.25) below.

Finally, we claim that there exists a modulus of continuity $\omega^{\prime}$ such that, for any $(y, Q, P) \in \mathscr{A}$,

$$
\left|\partial_{t} \mathcal{E}\left(t_{1}, y, Q, P\right)-\partial_{t} \mathcal{E}\left(t_{2}, y, Q, P\right)\right| \leq C\left(\mathcal{E}\left(t_{1}, y, Q, P\right)+1\right) \omega^{\prime}\left(t_{1}-t_{2}\right) .
$$

In particular, there also exists a modulus of continuity $\omega_{F}:[0, T] \rightarrow[0,+\infty)$ such that, for any $(y, Q, P) \in \mathscr{A}$,

$\mathcal{E}\left(t_{1}, y, Q, P\right) \leq F \Rightarrow\left|\partial_{t} \mathcal{E}\left(t_{1}, y, Q, P\right)-\partial_{t} \mathcal{E}\left(t_{2}, y, Q, P\right)\right| \leq \omega_{F}\left(t_{1}-t_{2}\right) \forall t_{2} \in[0, T]$.

Indeed, set

$$
A(t):=\mathbf{D} \mathcal{W}\left(P^{-1} Q^{T} \nabla \mathbf{g}(t, y) \nabla y\right)\left(P^{-1} Q^{T} \nabla \mathbf{g}(t, y) \nabla y\right)^{T},
$$

so that

$$
\partial_{t} \mathcal{E}(t, y, Q, P)=\int_{\Omega} A(t) \cdot P^{-1} Q^{T} \nabla \dot{\mathbf{g}}(t, y)(\nabla \mathbf{g}(t, y))^{-1} Q P d x .
$$

In view of (5.4), (5.5), for some constant $C>0$,

$$
\left\{\begin{array}{l}
\left\|\nabla \mathbf{g}\left(t_{1}, y\right)\left(\nabla \mathbf{g}\left(t_{1}, y\right)\right)^{-1}-\nabla \dot{\mathbf{g}}\left(t_{2}, y\right)\left(\nabla \mathbf{g}\left(t_{2}, y\right)\right)^{-1}\right\|_{L^{\infty}} \leq C\left|t_{1}-t_{2}\right| \\
\left\|\nabla \mathbf{g}\left(t_{2}, y\right)\left(\nabla \mathbf{g}\left(t_{1}, y\right)\right)^{-1}-I d\right\|_{L^{\infty}} \leq C\left|t_{1}-t_{2}\right|
\end{array}\right.
$$

By (5.1), (5.13), the following holds, for some constant $C>0$,

$$
\begin{aligned}
C \int_{\Omega}\left\{\left|A\left(t_{1}\right)-A\left(t_{2}\right)\right|+\left|A\left(t_{1}\right)\right| \mid\right. & \left.\nabla \dot{\mathbf{g}}\left(t_{1}, y\right)\left(\nabla \mathbf{g}\left(t_{1}, y\right)\right)^{-1}-\nabla \dot{\mathbf{g}}\left(t_{2}, y\right)\left(\nabla \mathbf{g}\left(t_{2}, y\right)\right)^{-1} \mid\right\} d x \\
\leq & C\left(\int_{\Omega}\left|A\left(t_{1}\right)-A\left(t_{2}\right)\right| d x+\int_{\Omega}\left|A\left(t_{1}\right)\right|\left|t_{1}-t_{2}\right| d x\right) .
\end{aligned}
$$

Using $\mathrm{H} 5$ we obtain

$$
\int_{\Omega}\left|A\left(t_{1}\right)\right|\left|t_{1}-t_{2}\right| d x \leq C\left(\mathcal{E}\left(\left(t_{1}, y, Q, P\right)\right)+1\right)\left|t_{1}-t_{2}\right| .
$$

The second inequality in (5.14), together with (5.1), leads to

$$
\left\|P^{-1} Q^{T}\left(\nabla \mathbf{g}\left(t_{2}, y\right)\left(\nabla \mathbf{g}\left(t_{1}, y\right)\right)^{-1}\right) Q P-I d\right\|_{L^{\infty}} \leq C\left|t_{1}-t_{2}\right| .
$$


Applying assumption $\mathrm{H} 6$ then yields the estimate

$$
\begin{aligned}
& \int_{\Omega}\left|A\left(t_{1}\right)-A\left(t_{2}\right)\right| d x \leq \\
& \begin{array}{r}
\omega\left(\left\|P^{-1} Q^{T} \nabla \mathbf{g}\left(t_{2}, y\right)\left(\nabla \mathbf{g}\left(t_{1}, y\right)\right)^{-1} Q P-I d\right\|_{L^{\infty}}\right)\left(\mathcal{E}\left(t_{1}, y, Q, P\right)+|\Omega|\right) \\
\leq \omega\left(C\left|t_{1}-t_{2}\right|\right)\left(\mathcal{E}\left(t_{1}, y, Q, P\right)+|\Omega|\right) .
\end{array}
\end{aligned}
$$

The claim (5.11) follows by inserting (5.16),(5.17) into (5.15) .

We now proceed to define a variational quasi-static evolution in the spirit of Proposition 4.1 and Definition 4.6. Recall that determinant terms drop out of the formulation and that the relevant definition of the dissipation is that of Remark 4.7 .

Definition 5.4. A variational quasi-static evolution for the boundary datum $t \mapsto$ $\mathbf{g}(t)$ is a function

$$
t \in[0, T] \mapsto(y(t), Q(t), P(t)) \in \mathscr{A}
$$

which satisfies the following conditions with $L(t):=\log P(t)$ :

(Global Minimality) For every $t \in[0, T]$

$$
\begin{aligned}
\mathcal{F}(t, y(t), Q(t), P(t)) \leq \mathcal{F}\left(t, y^{\prime}, Q^{\prime}, P^{\prime}\right)+\int_{\Omega} \mathcal{H}\left(L^{\prime}-L(t)\right) d x, & \\
& \forall\left(y^{\prime}, Q^{\prime}, P^{\prime}\right) \in \mathscr{A}, L^{\prime}:=\log P^{\prime} ;
\end{aligned}
$$

(Energy Conservation) For every $t \in[0, T]$

$$
\begin{aligned}
& \qquad \mathcal{F}(t, y(t), Q(t), P(t))+\operatorname{Diss}_{\mathcal{H}}(0, t ; L)=\mathcal{F}\left(0, y^{0}, Q^{0}, P^{0}\right)+ \\
& \int_{0}^{t} \int_{\Omega} \mathbf{D} \mathcal{W}\left(P^{-1}(s) Q^{T}(s) \nabla \mathbf{g}(s, y(s)) \nabla y(s)\right) \cdot P^{-1}(s) Q^{T}(s) \nabla \dot{\mathbf{g}}(s, y(s)) \nabla y(s) d x d s, \\
& \text { where } \\
& (5.18) \\
& \operatorname{Diss}_{\mathcal{H}}\left(t_{1}, t_{2} ; L\right):=\sup \left\{\sum_{i=1}^{N} \int_{\Omega} \mathcal{H}\left(L\left(s_{i}\right)-L\left(s_{i-1}\right)\right) d x: s_{0}=t_{1}<s_{1}<\ldots<s_{N}=t_{2}\right\} .
\end{aligned}
$$

The following existence result for a variational quasi-static evolution then holds true.

Theorem 5.5. Let $\left(y^{0}, Q^{0}, P^{0}\right) \in \mathscr{A}$ be a stable initial condition, that is that it satisfies

$$
\mathcal{F}\left(0, y_{0}, Q_{0}, P_{0}\right) \leq \mathcal{F}\left(0, y^{\prime}, Q^{\prime}, P^{\prime}\right)+\int_{\Omega} \mathcal{H}\left(L^{\prime}-L_{0}\right) d x
$$

for every $\left(y^{\prime}, Q^{\prime}, P^{\prime}\right) \in \mathscr{A}$ with $L_{0}:=\log P_{0}$. Then, there exists a variational quasistatic evolution

$$
t \mapsto(y(t), Q(t), P(t))
$$

such that

$$
y(0)=y_{0}, \quad Q(0)=Q_{0}, \quad P(0)=P_{0} \quad \text { and } L(0)=L_{0} .
$$

Above $\mathscr{A}$ is the set defined in (5.7). 
Remark 5.6. The existence of a stable initial condition in the sense of (5.19) will be established in Lemma 5.7 below.

5.1. An auxiliary problem. Our proof, as is customary by now, is based on a time incremental procedure. We first establish the existence of a globally stable condition for a problem which will provide for the existence of both an initially stable configuration, that is one that satisfies (5.19), and a time incremental solution.

Lemma 5.7. For every $t \in[0, T]$ and for every $L_{i n} \in L^{\infty}\left(\Omega ; \mathbb{M}^{3 \times 3}\right)$, there exists an element $(\bar{y}, \bar{Q}, \bar{P}) \in \mathscr{A}$ which minimizes

$$
\mathcal{F}(t, y, Q, P)+\int_{\Omega} \mathcal{H}\left(\log P-L_{i n}\right) d x
$$

over $\mathscr{A}$.

Proof. Let $\left\{\left(y^{n}, Q^{n}, P^{n}\right)\right\}$ be a minimizing sequence for

$$
\mathcal{F}(t, y, Q, P)+\int_{\Omega} \mathcal{H}\left(L-L_{i n}\right) d x
$$

over $\mathscr{A}$. Taking $\left(i d, I d, 1_{3}\right)$ as test field we obtain the following uniform bound

$$
\mathcal{F}\left(t, y^{n}, Q^{n}, P^{n}\right)+\int_{\Omega} \mathcal{H}\left(L^{n}-L_{i n}\right) d x \leq C
$$

by $\mathrm{H} 1$ and (5.3).

In view of the expression (5.8) for the energy, (5.1), (5.21) imply the existence of a constant $C$ such that

$$
\left\|\left(P^{n}\right)^{-1}\right\|_{L^{\infty}}+\left\|P^{n}\right\|_{L^{\infty}}+\left\|\nabla P^{n}\right\|_{L^{p}}+\left\|\nabla Q^{n}\right\|_{L^{p}} \leq C .
$$

Therefore, up to subsequences, there exists $(P, Q) \in W^{1, p}\left(\Omega ; \mathbb{R}_{1}^{3} \times S O(3)\right)$ such that

$$
\left(P^{n}, Q^{n}\right) \rightarrow(P, Q) \text { weakly in } W^{1, p}\left(\Omega ; \mathbb{R}_{1}^{3} \times S O(3)\right),
$$

so that

$$
\int_{\Omega}|\nabla P|^{p} d x+\int_{\Omega}|\nabla Q|^{p} d x \leq \liminf _{n \rightarrow+\infty} \int_{\Omega}\left\{\left|\nabla P^{n}\right|^{p}+\left|\nabla Q^{n}\right|^{p}\right\} d x \leq C .
$$

Since $p>3$, Rellich's theorem implies that

$$
\left(P^{n}, Q^{n}\right) \rightarrow(P, Q) \quad \text { strongly in } C^{0}\left(\bar{\Omega} ; \mathbb{R}_{1}^{3} \times S O(3)\right),
$$

so that, in particular, in view of the continuous character of $\widetilde{\mathcal{W}}_{\text {hard }}$,

$$
\mathcal{W}_{\text {hard }}\left(P^{n}\right) \rightarrow \mathcal{W}_{\text {hard }}(P) \text {, strongly in } C^{0}(\bar{\Omega}) .
$$

Moreover, since $L^{n}=\log P^{n}$, setting $L=\log P$, we conclude that

$$
\left(\left(P^{n}\right)^{-1}, L^{n}\right) \rightarrow\left(P^{-1}, L\right) \text { strongly in } C^{0}\left(\bar{\Omega} ; \mathbb{R}_{1}^{3} \times \mathbb{R}^{3}\right) .
$$

It is then immediate that

$$
\int_{\Omega} \mathcal{H}\left(L-L_{i n}\right) d x=\lim _{n \rightarrow+\infty} \int_{\Omega} \mathcal{H}\left(L^{n}-L_{\text {in }}\right) d x .
$$

We now investigate the lower semicontinuity of the elastic energy. Set $\varphi^{n}:=$ $\mathbf{g}\left(t, y^{n}\right)$. By $\mathrm{H} 4$ and (5.21),

$$
\left\|\operatorname{dist}\left(\left(P^{n}\right)^{-1}\left(Q^{n}\right)^{T} \nabla \varphi^{n}, S O(3)\right)\right\|_{L^{p}} \leq C \quad \text { for every } n \in \mathbb{N} .
$$


Therefore, (5.22) implies that

$$
\begin{aligned}
\left\|\nabla \varphi^{n}\right\|_{L^{p}} \leq C\left\|P^{n}\right\|_{L_{\infty}}\left\|Q^{n}\right\|_{L_{\infty}}\left\|\left(P^{n}\right)^{-1}\left(Q^{n}\right)^{T} \nabla \varphi^{n}\right\|_{L^{p}} \\
\quad \leq C\left(1+\left\|\operatorname{dist}\left(\left(P^{n}\right)^{-1}\left(Q^{n}\right)^{T} \nabla \varphi^{n}, S O(3)\right)\right\|_{L^{p}}\right) \leq C .
\end{aligned}
$$

Since $\varphi^{n}(x)=\mathbf{g}(t, x)$ on $\Gamma$, Poincaré's inequality yields that $\left\{\varphi^{n}\right\}$ is uniformly bounded in $W^{1, p}\left(\Omega ; \mathbb{R}^{3}\right)$. Hence, there exists $\varphi \in W^{1, p}\left(\Omega ; \mathbb{R}^{3}\right)$ such that, up to subsequences,

$$
\begin{cases}\varphi^{n} \rightarrow \varphi, & \text { weakly in } W^{1, p}\left(\Omega ; \mathbb{R}^{3}\right) \\ \varphi^{n} \rightarrow \varphi, & \text { strongly in } C^{0}\left(\bar{\Omega} ; \mathbb{R}^{3}\right),\end{cases}
$$

with $\varphi(x)=\mathbf{g}(t, x)$ on $\Gamma$. In turn, by (5.4), (5.5) and the analogue of (5.6) for $g^{-1}(t,$.$) , this implies that$

$$
\exists y \in \mathscr{Y} \text { such that } \varphi=\mathbf{g}(t, y) .
$$

Now, $\mathcal{W}$ is polyconvex, so that $\mathcal{W}(F)=h(F, \operatorname{cof} F, \operatorname{det} F)$ for some convex function $h: \mathbb{R}^{19} \rightarrow \overline{\mathbb{R}}$. By (5.28) and upon invoking the weak lower continuity for minors, we deduce that

$$
\operatorname{cof} \nabla \varphi^{n} \rightarrow \operatorname{cof} \nabla \varphi, \quad \text { weakly in } L^{\frac{p}{2}}\left(\Omega ; \mathbb{M}^{3 \times 3}\right)
$$

and

$\operatorname{det}\left[\left(P^{n}\right)^{-1}\left(Q^{n}\right)^{T} \nabla \varphi^{n}\right]=\operatorname{det} \nabla \varphi^{n} \rightarrow \operatorname{det}\left[P^{-1} Q^{T} \nabla \varphi\right]=\operatorname{det} \nabla \varphi, \quad$ weakly in $L^{\frac{p}{3}}(\Omega)$.

Now, by (5.24), (5.26) and (5.28) we obtain

$$
\left(P^{n}\right)^{-1}\left(Q^{n}\right)^{T} \nabla \varphi^{n} \rightarrow P^{-1} Q^{T} \nabla \varphi, \quad \text { weakly in } L^{p}\left(\Omega ; \mathbb{M}^{3 \times 3}\right) .
$$

Moreover,

$$
\begin{aligned}
\operatorname{cof}\left\{\left(P^{n}\right)^{-1}\left(Q^{n}\right)^{T} \nabla \varphi^{n}\right\} & =\operatorname{det}\left\{\left(P^{n}\right)^{-1}\left(Q^{n}\right)^{T} \nabla \varphi^{n}\right\}\left(\left(P^{n}\right)^{-1}\left(Q^{n}\right)^{T} \nabla \varphi^{n}\right)^{-T} \\
& =\left(\operatorname{det} \nabla \varphi^{n}\right) P^{n}\left(Q^{n}\right)^{T}\left(\nabla \varphi^{n}\right)^{-T}=P^{n}\left(Q^{n}\right)^{T} \operatorname{cof} \nabla \varphi^{n},
\end{aligned}
$$

hence by $(5.24),(5.26)$ and $(5.30)$,

$$
\operatorname{cof}\left\{\left(P^{n}\right)^{-1}\left(Q^{n}\right)^{T} \nabla \varphi^{n}\right\} \rightarrow P Q^{T} \operatorname{cof} \nabla \varphi=\operatorname{cof}\left\{(P)^{-1}(Q)^{T} \nabla \varphi\right\},
$$

weakly in $L^{\frac{p}{2}}\left(\Omega ; \mathbb{M}^{3 \times 3}\right)$.

By $(5.30),(5.31),(5.32)$, and because of the polyconvex character of $\mathcal{W}$, we conclude that

$$
\int_{\Omega} \mathcal{W}\left(P^{-1} Q^{T} \nabla \varphi\right) d x \leq \liminf _{n \rightarrow+\infty} \int_{\Omega} \mathcal{W}\left(\left(P^{n}\right)^{-1}\left(Q^{n}\right)^{T} \nabla \varphi^{n}\right) d x .
$$

Together, (5.6), (5.27), (5.29), (5.23), (5.25) and (5.33) imply the minimality of $(y, Q, P) \in \mathscr{A}$ for

$$
\mathcal{F}(t, y, Q, P)+\int_{\Omega} \mathcal{H}\left(L-L_{i n}\right) d x
$$


Lemma 5.7 is firstly applied to $L_{i n}=0$, yielding a triplet $\left(y_{0}, Q_{0}, P_{0}\right) \in \mathscr{A}$ that minimizes

$$
\mathcal{F}\left(0, y^{\prime}, Q^{\prime}, P^{\prime}\right)+\int_{\Omega} \mathcal{H}\left(L^{\prime}\right) d x,
$$

hence, because of the sub-additive character of $\mathcal{H}$, also such that it minimizes

$$
\mathcal{F}\left(0, y^{\prime}, Q^{\prime}, P^{\prime}\right)+\int_{\Omega} \mathcal{H}\left(L^{\prime}-L_{0}\right) d x
$$

with $L_{0}:=\log P_{0}$. The existence of a triplet $\left(y_{0}, Q_{0}, P_{0}\right) \in \mathscr{A}$ satisfying (5.19) is ensured.

5.2. Proof of Theorem 5.5. The proof follows a scheme which is classical by now; see e.g. [9] in the setting of small strain elasto-plasticity or [26] in that of Mielke's finite plasticity model. First, a time incremental minimization procedure yields a piecewise constant in time sequence of minimizers. Then, a priori estimates on that sequence are obtained. With those estimates at hand, the limit in the time discretization parameter is defined. It is first shown to satisfy global minimality. Then an upper inequality on the energy is obtained. Finally, an approximation result of Lebesgue integrals by appropriate Riemann sums is used to prove the other energy inequality.

Remark 5.8. The specifics of the proof also call upon results obtained in [20] in the setting of hyperelasticity, so that our argument should be seen as a path that meanders between the approach used in e.g. [9] and the more abstract approach favored in [20, Sections 3, 5]. It also parallels the approach used in the proof of the existence result for a regularized version of Mielke's plasticity model (see [26, Theorem 3.1]) alluded to in Remark 4.9.

Throughout the proof, $L$ (with appropriate sub- or superscripts) will stand for $\log P$ (with the corresponding sub- or superscript).

Consider a sequence of subdivisions $\left(t_{k}^{i}\right)_{0 \leq i \leq k}$ of the interval $[0, T]$ with

$$
0=t_{k}^{0}<t_{k}^{1}<\cdots<t_{k}^{k-1}<t_{k}^{k}=T,
$$

and

$$
\lim _{k \rightarrow+\infty} \max _{1 \leq i \leq k}\left(t_{k}^{i}-t_{k}^{i-1}\right)=0 .
$$
of

Set $\left(y_{k}^{0}, Q_{k}^{0}, P_{k}^{0}\right)=\left(y_{0}, Q_{0}, P_{0}\right)$ and, for $k \geq 1$, define $\left(y_{k}^{i}, Q_{k}^{i}, P_{k}^{i}\right)$ as an element

$$
\operatorname{Argmin}\left\{\mathcal{F}\left(t_{k}^{i}, y^{\prime}, Q^{\prime}, P^{\prime}\right)+\int_{\Omega} \mathcal{H}\left(L^{\prime}-L_{k}^{i-1}\right) d x:\left(y^{\prime}, Q^{\prime}, P^{\prime}\right) \in \mathscr{A}\right\} .
$$

By virtue of Lemma 5.7, that set is not empty.

Once again, by sub-additivity, $\left(y_{k}^{i}, Q_{k}^{i}, P_{k}^{i}\right)$ also satisfies, for all $\left(y^{\prime}, Q^{\prime}, P^{\prime}\right) \in \mathscr{A}$,

$$
\mathcal{F}\left(t_{k}^{i}, y_{k}^{i}, Q_{k}^{i}, P_{k}^{i}\right) \leq \mathcal{F}\left(t_{k}^{i}, y^{\prime}, Q^{\prime}, P^{\prime}\right)+\int_{\Omega} \mathcal{H}\left(L^{\prime}-L_{k}^{i}\right) d x .
$$


Step 1- Discrete energy inequality: Take $\left(y_{k}^{i-1}, Q_{k}^{i-1}, P_{k}^{i-1}\right)$ as a test triplet in (5.35). Then, in view of (5.9),

$$
\begin{aligned}
(5.37) \mathcal{F}\left(t_{k}^{i}, y_{k}^{i}, Q_{k}^{i}, P_{k}^{i}\right)+\int_{\Omega} \mathcal{H}\left(L_{k}^{i}-L_{k}^{i-1}\right) d x \leq \mathcal{F}\left(t_{k}^{i}, y_{k}^{i-1}, Q_{k}^{i-1}, P_{k}^{i-1}\right)= \\
\mathcal{F}\left(t_{k}^{i-1}, y_{k}^{i-1}, Q_{k}^{i-1}, P_{k}^{i-1}\right)+\int_{t_{k}^{i-1}}^{t_{k}^{i}} \partial_{s} \mathcal{F}\left(s, y_{k}^{i-1}, Q_{k}^{i-1}, P_{k}^{i-1}\right) d s= \\
\mathcal{F}\left(t_{k}^{i-1}, y_{k}^{i-1}, Q_{k}^{i-1}, P_{k}^{i-1}\right)+\int_{t_{k}^{i-1}}^{t_{k}^{i}} \int_{\Omega}\left\{\mathbf{D} \mathcal{W}\left(\left(P_{k}^{i-1}\right)^{-1}\left(Q_{k}^{i-1}\right)^{T} \nabla \mathbf{g}\left(s, y_{k}^{i-1}\right) \nabla y_{k}^{i-1}\right) .\right. \\
\left.\left(P_{k}^{i-1}\right)^{-1}\left(Q_{k}^{i-1}\right)^{T} \nabla \dot{\mathbf{g}}\left(s, y_{k}^{i-1}\right) \nabla y_{k}^{i-1}\right\} d x d s .
\end{aligned}
$$

Consider now the piecewise constant interpolants

$$
y_{k}(t)=y_{k}^{i_{t}}, \quad Q_{k}(t)=Q_{k}^{i_{t}}, \quad P_{k}(t)=P_{k}^{i_{t}},
$$

where $i_{t}$ is the largest integer such that $t_{k}^{i_{t}} \leq t$ and set $t_{k}(t):=t_{k}^{i_{t}}$.

Iteration of (5.37) yields, in view of the definition (5.18) of the dissipation,

$$
\begin{aligned}
\mathcal{F}\left(t_{k}(t), y_{k}(t), Q_{k}(t), P_{k}(t)\right)+\operatorname{Diss\mathcal {H}}\left(0, t ; L_{k}\right) \leq \\
\mathcal{F}\left(0, y_{0}, Q_{0}, P_{0}\right)+\int_{0}^{t_{k}(t)} \int_{\Omega}\left\{\mathbf{D} \mathcal{W}\left(P_{k}^{-1}(s) Q_{k}^{T}(s) \nabla \mathbf{g}\left(s, y_{k}(s)\right) \nabla y_{k}(s)\right)\right. \\
\left.P_{k}^{-1}(s) Q_{k}^{T}(s) \nabla \dot{\mathbf{g}}\left(s, y_{k}(s)\right) \nabla y_{k}(s)\right\} d x d s \\
=\mathcal{F}\left(0, y_{0}, Q_{0}, P_{0}\right)+\int_{0}^{t_{k}(t)} \partial_{t} \mathcal{F}\left(s, y_{k}(s), Q_{k}(s), P_{k}(s)\right) d s
\end{aligned}
$$

We set

$$
\begin{aligned}
A_{k}(t):=\mathbf{D} \mathcal{W}\left(P_{k}^{-1}(t) Q_{k}^{T}(t) \nabla \mathbf{g}\left(t, y_{k}(t)\right) \nabla y_{k}(t)\right) \\
\quad\left(P_{k}^{-1}(t) Q_{k}^{T}(t) \nabla \mathbf{g}\left(t, y_{k}(t)\right) \nabla y_{k}(t)\right)^{T},
\end{aligned}
$$

so that an equivalent expression for (5.9) is

$$
\begin{aligned}
& \partial_{t} \mathcal{F}\left(t, y_{k}(t), Q_{k}(t), P_{k}(t)\right)= \\
& \quad \int_{\Omega} A_{k}(t) \cdot P_{k}^{-1}(t) Q_{k}^{T}(t) \nabla \dot{\mathbf{g}}\left(t, y_{k}(t)\right)\left(\nabla \mathbf{g}\left(t, y_{k}(t)\right)\right)^{-1} Q_{k}(t) P_{k}(t) d x .
\end{aligned}
$$

In view of (5.39), (5.38) reads as

$$
\begin{gathered}
\mathcal{F}\left(t_{k}(t), y_{k}(t), Q_{k}(t), P_{k}(t)\right)+\operatorname{Diss} \mathcal{H}\left(0, t ; L_{k}\right) \leq \mathcal{F}\left(0, y_{0}, Q_{0}, P_{0}\right)+ \\
\int_{0}^{t_{k}(t)} \int_{\Omega} A_{k}(s) \cdot P_{k}^{-1}(s) Q_{k}^{T}(s) \nabla \dot{\mathbf{g}}\left(s, y_{k}(s)\right)\left(\nabla \mathbf{g}\left(s, y_{k}(s)\right)\right)^{-1} Q_{k}(s) P_{k}(s) d x d s .
\end{gathered}
$$

Step 2- Uniform bounds and limit stability: In this step we deduce some uniform bounds on the piecewise constant interpolants and we study their compactness 
properties. To this aim we first notice that, by $\mathrm{H} 5,(5.1),(5.5)$,

$$
\begin{aligned}
& \left|\int_{\Omega} A_{k}(s) \cdot P_{k}^{-1}(s) Q_{k}^{T}(s) \nabla \dot{\mathbf{g}}\left(s, y_{k}(s)\right)\left(\nabla \mathbf{g}\left(s, y_{k}(s)\right)\right)^{-1} Q_{k}(s) P_{k}(s) d x\right| \leq \\
& C \int_{\Omega} A_{k}(s) d x \leq C c_{2}\left(\int_{\Omega} \mathcal{W}\left(P_{k}^{-1}(s) Q_{k}^{T}(s) \nabla \mathbf{g}\left(s, y_{k}(s)\right) \nabla y_{k}(s)\right) d x+|\Omega|\right)
\end{aligned}
$$

for some constant $C>0$. Since $t_{k}(t) \leq t,(5.40)$, (5.41) imply that, for some constant $C>0$,

$$
\mathcal{E}\left(t_{k}(t), y_{k}(t), Q_{k}(t), P_{k}(t)\right) \leq C\left(1+\int_{0}^{t} \mathcal{E}\left(s, y_{k}(s), Q_{k}(s), P_{k}(s)\right) d s+T\right)
$$

Appealing to (5.11),

$$
\begin{aligned}
& \left|\mathcal{E}\left(t_{k}(t), y_{k}(t), Q_{k}(t), P_{k}(t)\right)-\mathcal{E}\left(t, y_{k}(t), Q_{k}(t), P_{k}(t)\right)\right| \leq \\
& \qquad \int_{t_{k}(t)}^{t}\left|\partial_{t} \mathcal{E}\left(s, y_{k}(t), Q_{k}(t), P_{k}(t)\right)\right| d s \leq\left|\partial_{t} \mathcal{E}\left(t, y_{k}(t), Q_{k}(t), P_{k}(t)\right)\right|\left(t-t_{k}(t)\right)+ \\
& \qquad \int_{t_{k}(t)}^{t}\left|\partial_{t} \mathcal{E}\left(s, y_{k}(t), Q_{k}(t), P_{k}(t)\right)-\partial_{t} \mathcal{E}\left(t, y_{k}(t), Q_{k}(t), P_{k}(t)\right)\right| d s \leq \\
& \left(t-t_{k}(t)\right)\left\{\left|\partial_{t} \mathcal{E}\left(t, y_{k}(t), Q_{k}(t), P_{k}(t)\right)\right|+C\left(\mathcal{E}\left(t, y_{k}(t), Q_{k}(t), P_{k}(t)\right)+1\right) \omega^{\prime}\left(t-t_{k}(t)\right)\right\}, \\
& \text { so that, using }(5.13),(5.16) \text { with } y_{k}(t), Q_{k}(t), P_{k}(t) \text { in lieu of } y, Q, P, \text { we conclude } \\
& \text { that } \\
& \qquad\left|\mathcal{E}\left(t_{k}(t), y_{k}(t), Q_{k}(t), P_{k}(t)\right)-\mathcal{E}\left(t, y_{k}(t), Q_{k}(t), P_{k}(t)\right)\right| \leq \\
& \qquad C\left(\mathcal{E}\left(t, y_{k}(t), Q_{k}(t), P_{k}(t)\right)+1\right) \omega^{\prime \prime}\left(t-t_{k}(t)\right),
\end{aligned}
$$

for some modulus of continuity $\omega^{\prime \prime}$, or still that, for $k$ large enough (independently of $t \in[0, T])$,

$$
\mathcal{E}\left(t, y_{k}(t), Q_{k}(t), P_{k}(t)\right) \leq \frac{\left(\mathcal{E}\left(t_{k}(t), y_{k}(t), Q_{k}(t), P_{k}(t)\right)+C \omega^{\prime \prime}\left(t-t_{k}(t)\right)\right.}{1-C \omega^{\prime \prime}\left(t-t_{k}(t)\right)} .
$$

Inserting (5.42) into (5.43) leads, for some constant $C_{T}$ depending on $T$, to

$$
\mathcal{E}\left(t, y_{k}(t), Q_{k}(t), P_{k}(t)\right) \leq C_{T}\left(\int_{0}^{t} \mathcal{E}\left(s, y_{k}(s), Q_{k}(s), P_{k}(s)\right) d s+1\right),
$$

provided that $k \geq k_{T}$, for some $k_{T}$ only depending on $T$. Applying Gronwall's inequality, we obtain, for $t \in[0, T]$, the estimate

$$
\mathcal{E}\left(t, y_{k}(t), Q_{k}(t), P_{k}(t)\right)=\int_{\Omega} \mathcal{W}\left(\left(P_{k}(t)\right)^{-1}\left(Q_{k}(t)\right)^{T} \nabla \mathbf{g}\left(t, y_{k}(t)\right) \nabla y_{k}(t)\right) d x \leq C_{T},
$$

with $C_{T}$ possibly depending on $T$, but independent of $k \geq k_{T}$. Reapplying (5.40), (5.41), we conclude with the help of (5.44), of the expression for $\mathcal{F}$ in (5.8) and of (5.3) that there exists a possibly different constant $C_{T}$ depending on $T$, but independent of $k$ such that

$$
\mathcal{F}\left(t, y_{k}(t), Q_{k}(t), P_{k}(t), L_{k}(t)\right)+\operatorname{Var}\left(0, t ; L_{k}\right) \leq C_{T} .
$$

The bound on the total variation of $L_{k}$ in (5.45) permits application of a generalized version of Helly's Selection Principle [25, Theorem 3.2]. Thus, there exists 
a map $L \in B V\left([0, T] ; \mathcal{M}_{b}\left(\Omega ; \mathbb{R}_{1}^{3}\right)\right)$ such that, for a subsequence of $L_{k}$, still indexed by $k$,

$$
L_{k}(t) \rightarrow^{*} L(t) \text { weak }{ }^{*} \text { in } \mathcal{M}_{b}\left(\Omega ; \mathbb{R}_{1}^{3}\right) \text { for every } t \in[0, T] .
$$

Recalling (5.1),(5.5), we deduce, as in the proof of (5.28), the uniform bound

$$
\left\|P_{k}^{-1}(t)\right\|_{L^{\infty}}+\left\|y_{k}(t)\right\|_{W^{1, p}}+\left\|P_{k}(t)\right\|_{W^{1, p}}+\left\|Q_{k}(t)\right\|_{W^{1, p}} \leq C_{T} .
$$

Now fix $t \in[0, T]$. In view of (5.47), there exists a possibly $t$-dependent subsequence $\left\{\left(y_{k_{t}}(t), Q_{k_{t}}(t), P_{k_{t}}(t)\right)\right\}$, and a triplet $(y(t), Q(t), P(t)) \in \mathscr{A}$ such that

$$
\left\{\begin{array}{lll}
y_{k_{t}}(t) & \rightarrow y(t), & \text { weakly in } W^{1, p}\left(\Omega ; \mathbb{R}^{3}\right) \\
P_{k_{t}}(t) & \rightarrow P(t), & \text { weakly in } W^{1, p}\left(\Omega ; \mathbb{R}_{1}^{3}\right) \\
Q_{k_{t}}(t) & \rightarrow Q(t), & \text { weakly in } W^{1, p}(\Omega ; S O(3))
\end{array}\right.
$$

Since $p>3$, we also obtain the following convergences:

$$
\left\{\begin{array}{lll}
L_{k_{t}}(t) & \rightarrow L(t), & \text { strongly in } C^{0}\left(\bar{\Omega} ; \mathbb{R}_{1}^{3}\right) \\
y_{k_{t}}(t) & \rightarrow y(t), & \text { strongly in } C^{0}\left(\bar{\Omega} ; \mathbb{R}^{3}\right) .
\end{array}\right.
$$

Passing to the liminf in the left hand-side of (5.36) is achieved upon noting that $t_{k}(t){ }^{k} t$ and applying the lower semi-continuity result (5.10). In view of (5.9) (which implies continuity in $t$ of the funcional $\mathcal{E}\left(., y^{\prime}, Q^{\prime}, P^{\prime}\right)$ ) and of (5.48), passing to the limit in the right hand-side of (5.36) is immediate. Thus,

$$
\begin{aligned}
\mathcal{F}(t, y(t), Q(t), P(t)) \leq \liminf _{k_{t}} \mathcal{F}\left(t_{k_{t}}(t), y_{k_{t}}(t), Q_{k_{t}}(t), P_{k_{t}}(t)\right) \\
\quad \leq \mathcal{F}\left(t, y^{\prime}, Q^{\prime}, P^{\prime}\right)+\int_{\Omega} \mathcal{H}\left(L^{\prime}-L(t)\right) d x
\end{aligned}
$$

for all $\left(y^{\prime}, Q^{\prime}, P^{\prime}\right) \in \mathscr{A}$.

Finally, in view of (5.46), the convergence of $L_{k}(t)$ in (5.48) actually takes place for the whole sequence, that is

$$
L_{k}(t) \rightarrow L(t), \text { strongly in } C^{0}\left(\bar{\Omega} ; \mathbb{R}_{1}^{3}\right) .
$$

Consequently, recalling the definition (5.18) of the dissipation, it is straightforward that, for any $0 \leq t_{1} \leq t_{2} \leq T$,

$$
\operatorname{Diss}_{\mathcal{H}}\left(t_{1}, t_{2} ; L\right) \leq \liminf _{k} \operatorname{Diss}_{\mathcal{H}}\left(t_{1}, t_{2} ; L_{k}\right) .
$$

Step 3 - Convergence of the right-hand side of (5.38): We set

$$
\begin{aligned}
\theta_{k}(s):=\partial_{t} \mathcal{F}\left(s, y_{k}(s), Q_{k}(s), P_{k}(s)\right)=\partial_{t} \mathcal{E}\left(s, y_{k}(s), Q_{k}(s), P_{k}(s)\right)= \\
\quad \int_{\Omega} A_{k}(s) \cdot P_{k}^{-1}(s) Q_{k}^{T}(s) \nabla \dot{g}\left(s, y_{k}(s)\right)\left(\nabla \mathbf{g}\left(s, y_{k}(s)\right)\right)^{-1} Q_{k}(s) P_{k}(s) d x .
\end{aligned}
$$

Combining (5.41) and (5.44) we obtain that

$$
\left\|\theta_{k}(s)\right\|_{L^{\infty}(0, T)} \leq C .
$$

Define

$$
\theta(t):=\limsup _{k \rightarrow+\infty} \theta_{k}(t)
$$


Fatou's lemma immediately implies that $\theta \in L^{1}(0, T)$ and that, since $t_{k}(t) \stackrel{k}{\nearrow} t$,

$$
\limsup _{k} \int_{0}^{t_{k}(t)} \theta_{k}(s) d s \leq \int_{0}^{t} \theta(s) d s .
$$

Further, we can extract a $t$-dependent subsequence $k_{t}$ such that

$$
\theta(t)=\lim _{k_{t}} \theta_{k_{t}}(t)
$$

The $t$-dependent subsequences extracted at the end of the previous step will be taken to be subsequences of $\left\{k_{t}\right\}$ that we will not relabel.

We now show that

$$
\theta(t)=\partial_{t} \mathcal{F}(t, y(t), Q(t), P(t))=\partial_{t} \mathcal{E}(t, y(t), Q(t), P(t)) .
$$

To this effect, we first remark that inserting the test triplet $\left(y(t), Q_{k_{t}}^{i}, P_{k_{t}}^{i}\right) \in \mathscr{A}$ into (5.36) yields

$$
\begin{aligned}
\int_{\Omega} \mathcal{W}\left(P_{k_{t}}^{-1}(t) Q_{k_{t}}^{T}(t) \nabla \mathbf{g}\left(t_{k}(t), y_{k_{t}}(t)\right) \nabla y_{k_{t}}(t)\right) d x \leq \\
\int_{\Omega} \mathcal{W}\left(P_{k_{t}}^{-1}(t) Q_{k_{t}}^{T}(t) \nabla \mathbf{g}\left(t_{k}(t), y(t)\right) \nabla y(t)\right) d x .
\end{aligned}
$$

Hence, because of H1, (5.5) and the uniform convergence of $P_{k_{t}}(t)$ and $Q_{k_{t}}(t)$, Fatou's lemma implies

$$
\begin{aligned}
\underset{k}{\limsup } \int_{\Omega} \mathcal{W}\left(P_{k_{t}}^{-1}(t) Q_{k_{t}}^{T}(t) \nabla \mathbf{g}\left(t_{k_{t}}(t), y_{k_{t}}(t)\right) \nabla y_{k_{t}}(t)\right) d x \leq \\
\int_{\Omega} \mathcal{W}\left(P^{-1}(t) Q^{T}(t) \nabla \mathbf{g}(t, y(t)) \nabla y(t)\right) d x .
\end{aligned}
$$

As when deriving (5.49), passing to the liminf in the integral in the left hand-side of the above inequality is achieved upon noting that $t_{k}(t){ }^{k} \nearrow t$ and applying the lower semi-continuity result (5.10). We get

$$
\begin{aligned}
\int_{\Omega} \mathcal{W}\left(P^{-1}(t) Q^{T}(t)\right. & \nabla \mathbf{g}(t, y(t)) \nabla y(t)) d x \\
\leq \liminf _{k_{t} \rightarrow+\infty} & \int_{\Omega} \mathcal{W}\left(P_{k_{t}}^{-1}(t) Q_{k_{t}}^{T}(t) \nabla \mathbf{g}\left(t_{k_{t}}(t), y_{k_{t}}(t)\right) \nabla y_{k_{t}}(t)\right) d x \\
\leq & \limsup _{k_{t} \rightarrow+\infty} \int_{\Omega} \mathcal{W}\left(P_{k_{t}}^{-1}(t) Q_{k_{t}}^{T}(t) \nabla \mathbf{g}\left(t_{k_{t}}(t), y_{k_{t}}(t)\right) \nabla y_{k_{t}}(t)\right) d x .
\end{aligned}
$$

Then, we infer from (5.55) that

$$
\begin{aligned}
\lim _{k_{t} \rightarrow+\infty} \int_{\Omega} \mathcal{W}\left(P_{k_{t}}^{-1}(t) Q_{k_{t}}^{T}(t) \nabla \mathbf{g}\left(t_{k_{t}}(t), y_{k_{t}}(t)\right) \nabla y_{k_{t}}(t)\right) d t= \\
\int_{\Omega} \mathcal{W}\left(P^{-1}(t) Q^{T}(t) \nabla \mathbf{g}(t, y(t)) \nabla y(t)\right) d t .
\end{aligned}
$$

In view of (5.56), and because of the uniform continuity property (5.12), we are at liberty to apply [20, Proposition 3.3]. We conclude to the continuity under weak convergence of $\partial_{t} \mathcal{E}(t, y, P, Q)$, which establishes (5.54). 
Step 4 - Energy Conservation: First,

$$
\begin{aligned}
\mathcal{F}(t, y(t), Q(t), P(t))+\operatorname{Diss}_{\mathcal{H}}(0, t & ; L) \\
\leq \mathcal{F}\left(0, y^{0}, Q^{0}, P^{0}\right)+\int_{0}^{t} \int_{\Omega} \mathbf{D} \mathcal{W}( & \left(P^{-1}(t)(Q)^{T}(t) \nabla \mathbf{g}(t, y(t)) \nabla y(t)\right) \\
\cdot & \left.P^{-1}(t)(Q)^{T}(t) \nabla \dot{\mathbf{g}}(t, y(t)) \nabla y(t)\right\} d x d s .
\end{aligned}
$$

Indeed, (5.51) and the first inequality in (5.49) permit to pass to the liminf in (5.38) yielding

$$
\begin{aligned}
\mathcal{F}(t, y(t), Q(t), P(t))+\operatorname{Diss}_{\mathcal{H}}( & 0, t ; L) \leq \mathcal{F}\left(0, y_{0}, Q_{0}, P_{0}\right) \\
& +\liminf _{k_{t} \rightarrow \infty} \int_{0}^{t_{k_{t}}(t)} \partial_{t} \mathcal{F}\left(s, y_{k_{t}}(s), Q_{k_{t}}(s), P_{k_{t}}(s)\right) d s .
\end{aligned}
$$

Inequality (5.57) is obtained upon recalling (5.52), (5.54) and (5.9).

We now address the other energy inequality. Fix $t \in[0, T]$ and let $\left(s_{k}^{i}\right)_{0 \leq i \leq k}$ be a sequence of subdivisions of $[0, t]$ such that

$$
0=s_{k}^{0}<s_{k}^{1}<\cdots<s_{k}^{k}=t
$$

and

$$
\lim _{k \rightarrow+\infty} \max _{0 \leq i \leq k}\left(s_{k}^{i}-s_{k}^{i-1}\right)=0 .
$$

Application of (5.49) leads to

$$
\begin{gathered}
\left.\mathcal{F}\left(s_{k}^{i-1}, y\left(s_{k}^{i-1}\right), Q\left(s_{k}^{i-1}\right), P\left(s_{k}^{i-1}\right)\right)\right) \leq \mathcal{F}\left(s_{k}^{i-1}, y\left(s_{k}^{i}\right), Q\left(s_{k}^{i}\right), P\left(s_{k}^{i}\right)\right)+ \\
\int_{\Omega} \mathcal{H}\left(L\left(s_{k}^{i}\right)-L\left(s_{k}^{i-1}\right)\right) d x=\mathcal{F}\left(s_{k}^{i}, y\left(s_{k}^{i}\right), Q\left(s_{k}^{i}\right), P\left(s_{k}^{i}\right)\right)+ \\
\int_{\Omega} \mathcal{H}\left(L\left(s_{k}^{i}\right)-L\left(s_{k}^{i-1}\right)\right) d x-\int_{s_{k}^{i-1}}^{s_{k}^{i}} \partial_{t} \mathcal{F}\left(s, y\left(s_{k}^{i}\right), Q\left(s_{k}^{i}\right), P\left(s_{k}^{i}\right)\right) d s .
\end{gathered}
$$

Consider now the piecewise constant interpolants

$$
y_{k}(s)=y\left(s_{k}^{i}\right), Q_{k}(s)=Q\left(s_{k}^{i}\right), P_{k}(s)=P\left(s_{k}^{i}\right) \text { and } L_{k}(s)=L\left(s_{k}^{i}\right),
$$

where $i$ is the smallest integer such that $s \leq s_{k}^{i}$. Since $s_{k}^{k}=t$, iterating (5.59) leads to

$$
\begin{aligned}
& \sum_{i=1}^{k} \int_{s_{k}^{i-1}}^{s_{k}^{i}} \partial_{t} \mathcal{F}\left(s, y\left(s_{k}^{i}\right), Q\left(s_{k}^{i}\right), P\left(s_{k}^{i}\right)\right) d s \leq \\
& \sum_{i=1}^{k} \int_{\Omega} \mathcal{H}\left(L\left(s_{k}^{i}\right)-L\left(s_{k}^{i-1}\right)\right) d x+\mathcal{F}\left(t, y_{k}(t), Q_{k}(t), P_{k}(t)\right)-\mathcal{F}\left(0, y^{0}, Q^{0}, P^{0}\right) \leq \\
& \operatorname{Diss}_{\mathcal{H}}(0, t ; L)+\mathcal{F}\left(t, y_{k}(t), Q_{k}(t), P_{k}(t)\right)-\mathcal{F}\left(0, y^{0}, Q^{0}, P^{0}\right) .
\end{aligned}
$$

Now,

$$
\limsup _{k \rightarrow+\infty} \sum_{i=0}^{k} \int_{s_{k}^{i-1}}^{s_{k}^{i}} \partial_{t} \mathcal{F}\left(s, y\left(s_{k}^{i}\right), Q\left(s_{k}^{i}\right), P\left(s_{k}^{i}\right)\right) d s=\int_{0}^{t} \partial_{t} \mathcal{F}(s, y(s), Q(s), P(s)) d s
$$


The proof of (5.60) adapts an argument developed in [20, Step 5, Proof of Theorem 3.4]. Taking $\left(i d, I d, 1_{3}\right)$ as test field in the stability condition (5.49) and recalling (5.1), (5.3) yields the existence of a constant $C$ such that

$$
\mathcal{F}(t, y(t), Q(t), P(t)) \leq C \quad \text { for every } t \in[0, T] .
$$

Moreover,

$$
\begin{aligned}
\sum_{i=0}^{k} \int_{s_{k}^{i-1}}^{s_{k}^{i}} \partial_{s} \mathcal{F}\left(s, y\left(s_{k}^{i}\right), Q\left(s_{k}^{i}\right), P\left(s_{k}^{i}\right)\right) d s= \\
\sum_{i=0}^{k}\left(s_{k}^{i}-s_{k}^{i-1}\right) \partial_{s} \mathcal{F}\left(s_{k}^{i}, y\left(s_{k}^{i}\right), Q\left(s_{k}^{i}\right), P\left(s_{k}^{i}\right)\right) d s-\sum_{i=0}^{k} \rho_{k}^{i},
\end{aligned}
$$

where

$$
\rho_{k}^{i}:=\int_{s_{k}^{i-1}}^{s_{k}^{i}}\left\{\partial_{s} \mathcal{F}\left(s, y\left(s_{k}^{i}\right), Q\left(s_{k}^{i}\right), P\left(s_{k}^{i}\right)\right)-\partial_{s} \mathcal{F}\left(s_{k}^{i}, y\left(s_{k}^{i}\right), Q\left(s_{k}^{i}\right), P\left(s_{k}^{i}\right)\right)\right\} d s .
$$

In the light of (5.61), (5.12) applies, hence

$$
\left|\rho_{k}^{i}\right| \leq\left(s_{k}^{i}-s_{k}^{i-1}\right) \omega_{C}\left(s_{k}^{i}-s_{k}^{i-1}\right) \leq\left(s_{k}^{i}-s_{k}^{i-1}\right) \omega_{C}\left(\max _{0 \leq i \leq k}\left(s_{k}^{i}-s_{k}^{i-1}\right)\right),
$$

or still, because of (5.58),

$$
\begin{aligned}
\underset{k}{\limsup } \sum_{i=0}^{k} \int_{s_{k}^{i-1}}^{s_{k}^{i}} \partial_{s} \mathcal{F}\left(s, y\left(s_{k}^{i}\right), Q\left(s_{k}^{i}\right), P\left(s_{k}^{i}\right)\right) d s= \\
\quad \underset{k}{\limsup } \sum_{i=0}^{k}\left(s_{k}^{i}-s_{k}^{i-1}\right) \partial_{s} \mathcal{F}\left(s_{k}^{i}, y\left(s_{k}^{i}\right), Q\left(s_{k}^{i}\right), P\left(s_{k}^{i}\right)\right) d s .
\end{aligned}
$$

Since $s \mapsto \partial_{t} \mathcal{F}(s, y(s), Q(s), P(s)) \in L^{1}(0, T)$ by (5.54), equality (5.60) follows now by (5.58), thanks to an approximation result of Lebesgue integrals by appropriately chosen Riemann sums; see [11, Lemma 4.12].

The proof of Theorem 5.5 is complete.

\section{The RIGID-PLASTIC CASE}

As mentioned earlier, we are currently unable to propose a satisfactory functional framework for the rate independent evolution identified in Proposition 4.1. The usual approach to the existence proof consists - as illustrated e.g. in the proof of Theorem 5.5 above - in incrementing time and iteratively solving a finite number of minimization problems at each time increment. It might be tempting to further specialize the setting in the hope of securing at the least an existence result for the incremental process.

One such effort was initiated in [7], then pursued in e.g. [5, 6] in the setting of crystal plasticity already evoked in Remark 2.1. In that context the adopted multiplicative decomposition is taken to be $F=E P$, but $P$ is restricted to be of the form

$$
P=I+(F m \cdot F s) s \otimes m, s, m \text { fixed orthogonal unit vectors }
$$


(see [7, Equations (2.5), (2.8)]). In any case, the main simplification there is that elasticity is replaced by rigidity. In other words, the following frame indifferent free energy density is investigated:

$$
\mathcal{W}(E)= \begin{cases}0, & E \in S O(N) \\ \infty, & \text { else }\end{cases}
$$

Then, taking $F=I$ at the initial time, the authors strive to compute the infimum of the incremental problem, especially at the first time step for which the problem becomes

$$
\inf \left\{\int_{\Omega}|F s \cdot F m| d x: F=R(I+(F m \cdot F s)) s \otimes m, R \in S O(N)\right\} .
$$

Our purpose is not to describe their result, but rather to implement a similar scheme in the present setting.

We adopt (6.1) as energy, take, as they did, $F=I$ at the initial time and complete the model with the following Von-Mises type set of admissible stresses:

$$
\mathbf{K}:=\left\{|C| \leq c: C \in \mathbb{M}_{D}^{N \times N}\right\},
$$

or still

$$
\mathcal{H}(T)=c|T|, T \in \mathbb{M}_{D}^{N \times N} .
$$

Now, recalling that $E=P^{-1} Q^{T} F, \mathcal{W}(E)=\mathcal{W}\left(P^{-1} Q^{T} F\right)$ and thus $\mathcal{W}(E)=0$ if, and only if $P^{-1} Q^{T} F \in S O(N)$, or still if, and only if $F=Q P R, R \in S O(N)$, so that the diagonal entries of $P$ identify with the singular values of $F$.

Remark that the multiplicative decomposition of $F$ into $Q P E$ is certainly not uniquely determined for a given $F \in \mathbb{M}^{N \times N}$. However, we are at liberty to define the reduced energy

$$
\begin{aligned}
\hat{\mathcal{W}}(F):= & \min \left\{\int_{\Omega} \mathcal{W}(E)+\mathcal{H}(\log P) ; F=Q P E\right. \text { for some } \\
& Q \in S O(N), P \text { positive definite and diagonal with } \operatorname{det} P=1\} .
\end{aligned}
$$

In view of the above, $\hat{\mathcal{W}}$ is easily identified as

$$
\hat{\mathcal{W}}(F):= \begin{cases}c \sqrt{\sum_{i=1}^{N} \lambda_{i}^{2}}, & \left\{\lambda_{i}\right\} \text { singular values of } F, \operatorname{det} F=1 \\ \infty & \text { else. }\end{cases}
$$

We now specialize (6.4) to the case when $N=3$, so that

$$
\hat{\mathcal{W}}(F)=\left\{\begin{array}{c}
c \sqrt{\left|\log \lambda_{1}\right|^{2}+\left|\log \lambda_{2}\right|^{2}+\left|\log \lambda_{3}\right|^{2}}, \operatorname{det} F=1, \\
\quad \lambda_{1}, \lambda_{2}, \lambda_{3}=1 / \lambda_{1} \lambda_{2} \text { singular values of } F \\
\infty, \text { else. }
\end{array}\right.
$$

This can equivalently be written as

$$
\hat{\mathcal{W}}(F):=h(\operatorname{det} F)+c \sqrt{\left|\log \lambda_{1}\right|^{2}+\left|\log \lambda_{2}\right|^{2}+\left|\log \lambda_{3}\right|^{2}}, \text { with } h(g):= \begin{cases}0, & g=1 \\ \infty, & \text { else. }\end{cases}
$$

Note that $F \rightarrow h(\operatorname{det} F)$ is polyconvex. 
We wish, as in [7], to determine the lower semi-continuous envelope of

$$
\mathscr{W}: \varphi \rightarrow \int_{\Omega} \hat{\mathcal{W}}(\nabla \varphi) d x
$$

Of course, the topology for which lower semi-continuity is sought has to be specified beforehand. As stated several times before, the correct functional framework is unclear to us at present. We will restrict our attention to the weak topology of $W^{1, p}\left(\Omega ; \mathbb{R}^{N}\right), 1 \leq p<\infty$, or the weak-* topology of $W^{1, \infty}\left(\Omega ; \mathbb{R}^{N}\right)$, thereby neglecting any kind of concentration effect. Then, the following lemma holds true:

Lemma 6.1 (The 3d rigid-plastic case). Assume that the lower semi-continuous envelope of $\mathscr{W}$ is local, i.e., of the form

$$
\varphi \rightarrow \int_{\Omega} Q \hat{\mathcal{W}}(\nabla \varphi) d x
$$

for some $Q \hat{\mathcal{W}}: \mathbb{M}^{N \times N} \rightarrow \overline{\mathbb{R}}$. Then,

$$
Q \hat{\mathcal{W}}(F) \equiv 0, \text { if } \operatorname{det} F=1 .
$$

Proof. According to [1, Corollary 3.2], locality of the lower semi-continuous envelope of $\mathscr{W}$ on $W^{1, p}\left(\Omega ; \mathbb{R}^{N}\right)$ implies that $Q \hat{\mathcal{W}}$ is $W^{1, p}$-quasiconvex. Then, from [37, P.164], $Q \hat{\mathcal{W}}$ is in particular rank-1 convex.

For instance $t \rightarrow Q \hat{\mathcal{W}}\left(M+t e_{1} \otimes e_{3}\right)$ must be convex in $t$ for every $M \in \mathbb{R}^{3 \times 3}$. Take

with $a, b>0$ and consider the map

$$
M=\left(\begin{array}{ccc}
a & 0 & 0 \\
0 & b & 0 \\
0 & 0 & \frac{1}{a b}
\end{array}\right)
$$

$$
M(t)=M+t \vec{e}_{1} \otimes \vec{e}_{3}
$$

which satisfies $\operatorname{det} M(t)=1$. The singular values of $M(t)$ are (6.6)

$$
\begin{aligned}
& \lambda_{1}=b \\
& \lambda_{2}=\sqrt{\frac{t^{2}+1 / a^{2} b^{2}+a^{2}+t^{2} \sqrt{1+1 / t^{4}\left(1 / a^{2} b^{2}+a^{2}\right)^{2}+2 / t^{2}\left(1 / a^{2} b^{2}+a^{2}\right)-4 / t^{4} b^{2}}}{2}} \\
& \lambda_{3}=\frac{1}{b \lambda_{2}} .
\end{aligned}
$$

Consequently,

$$
\begin{aligned}
& \hat{\mathcal{W}}(M(t))=c \sqrt{\left(\log \lambda_{1}\right)^{2}+\left(\log \lambda_{2}\right)^{2}+\left(\log \lambda_{3}\right)^{2}} \\
& =c \sqrt{(\log b)^{2}+\left(\log \lambda_{2}\right)^{2}+\left(\log b+\log \lambda_{2}\right)^{2}} \\
& =c \sqrt{2(\log b)^{2}+2\left(\log \lambda_{2}\right)^{2}+2 \log b \log \lambda_{2}} \\
& =c \sqrt{2}\left|\log \lambda_{2}\right| \sqrt{1+\frac{(\log b)^{2}}{\left(\log \lambda_{2}\right)^{2}}+\frac{\log b}{\log \lambda_{2}}} .
\end{aligned}
$$


So, for some $C>0$,

$$
0 \leq Q \hat{\mathcal{W}}(M(t)) \leq \hat{\mathcal{W}}(M(t)) \leq C \log t, \text { as } t \nearrow+\infty .
$$

But a non negative convex function with sublinear growth at $\infty$ must be identically null. We conclude that

$$
Q \hat{\mathcal{W}}(M(t))=0 .
$$

Since this holds independently of $(a, b) \in \mathbb{R}^{2}$ and of $t \in(0, \infty), \lambda_{2}$ given in (6.6) varies between 0 and $\infty$. All possible singular values of an arbitrary $F$ with $h(\operatorname{det} F)=0$ are spanned. Summing up,

$$
0 \leq Q \hat{\mathcal{W}}(F) \leq h(\operatorname{det} F)
$$

so that the lower semi-continuous envelope of $\mathscr{W}$ is trivial, at least as far as incompressible deformations are concerned.

Remark 6.2. A similar but simpler argument would demonstrate that the analogue of Lemma 6.1 also holds true when the dimension $N$ is 2 .

The conclusion of Lemma 6.1 delivers a serious blow to our newly formulated model of finite elasto-plasticity and would, at first glance, militate for a different model. Maybe so, but, in all fairness, the kind of pathology which we have just observed is not a specificity of our model. It will occur in all models of finite plasticity that are based on a multiplicative decomposition of the total deformation gradient, and this independently of the order of the decomposition, provided that the dissipation involves the product $P^{-1} \dot{P}$, or else $\dot{P} P^{-1}$ because this will inevitably produce a dissipation functional with some kind of logarithmic growth.

Remark 6.3. In particular, the model introduced in Remark 4.9 will produce a similar result. Indeed, taking as we $\operatorname{did} F=I$ at the initial time, the dissipation $H(\log P)$ in our model $(6.3)$ is replaced by $\mathcal{D}(I, P)$ defined in (4.11).

Now, for those $P$ 's for which the real logarithm of $P$ can be defined unambiguously, insertion of $P(s):=\exp (s \log P), s \in[0,1]$ into the expression for $\mathcal{D}(I, P)$ immediately yields

$$
\mathcal{D}(I, P) \leq \int_{0}^{1} \mathcal{H}\left(P^{-1}(s) \dot{P}(s)\right) d s=\mathcal{H}(\log P) .
$$

The lack of a universal formula for the real logarithm of a matrix when it exists (see for example [8]) and our willingly conceded lack of agility in that topic, makes it difficult for us to conclude in full generality as we did in Lemma 6.1.

Consequently, we content ourselves with the following example. Consider the unimodular matrix

$$
N_{a, b}=\left(\begin{array}{rcc}
0 & a & 0 \\
-b & 0 & 0 \\
0 & 0 & 1 /(a b)
\end{array}\right)
$$

Then

$$
N_{a, b}(t):=N_{a, b}+t \vec{e}_{1} \otimes \vec{e}_{1}=\left(\begin{array}{rcc}
t & a & 0 \\
-b & 0 & 0 \\
0 & 0 & 1 /(a b)
\end{array}\right)
$$


is a rank one perturbation of $N_{a, b}$. It eigenvalues, for $t \geq 2 \sqrt{a b}$, are $-1 / 2\left(\sqrt{t^{2}-4 a b}-\right.$ $t), 1 / 2\left(\sqrt{t^{2}-4 a b}+t\right), 1 /(a b)$, so that it is diagonalizable. Its logarithm is easily computed to be

$$
\left(\begin{array}{ccc}
-\frac{1}{2 \sqrt{t^{2}-4 a b}}[(t- & 2 a \\
\left.\sqrt{t^{2}-4 a b}\right) \log (a b) & \frac{}{\sqrt{t^{2}-4 a b}} \log (t+ & \\
\frac{+2 t \log (t-}{\left.\sqrt{t^{2}-4 a b}\right)} & \\
\left.\sqrt{t^{2}-4 a b}-t \log 4\right] & -\log (4 a b) & \\
& \frac{a}{2 \sqrt{t^{2}-4 a b}}[-(t+ & \\
\frac{b}{\sqrt{t^{2}-4 a b}} \log (a b) & +2 t \log \left(t-\sqrt{t^{2}-4 a b}\right) & \\
& -t \log 4] & \\
0 & 0 & -\frac{a}{\sqrt{t^{2}-4 a b}} \log (a b)
\end{array}\right)
$$

so that an argument identical to that leading to (6.7) would show that the lower semi-continuous envelope of the rigid-plastic energy would be identically 0 on the set of matrices defined as $\left\{N_{a, b}(t): a>0, b>0, t \geq 2 \sqrt{a b}\right\}$. We conjecture that the full force of Lemma 6.1 also holds true in Mielke's setting.

The model of crystal plasticity invoked in [7] is different to the extent that the slip directions that can be activated (the possible plastic strains $P$ ) are severely constrained (a single slip direction in the original contribution [7]). Such luxury is not available if considering a more general model of finite plasticity.

We venture to posit that the true culprit is the multiplicative decomposition which automatically creates a dissipation involving the ill-fated product.

\section{The one Dimensional Case - A GeneriC Study of STABility}

In this last section, we wish to further illustrate our discomfort with the multiplicative decomposition. In a one-dimensional setting all models of plasticity that abide by the multiplicative decomposition merge into a single model and there is hardly any room for rivalry between competing theories. Of course, in such a restrictive environment, the geometric constraints have to be relaxed and we cannot assume any longer that the plastic strain should be isochoric.

And yet, even such a simplistic setting carries the seeds of discontent. Indeed, decompose the deformation $\varphi^{\prime}$ into a plastic part $p>0$ and an elastic part $e$, namely,

$$
\varphi^{\prime}=e p=p e .
$$

The free energy $\mathcal{W}(e)$ is only function of $e$. In the footsteps of Section 3, it is immediate that the first Piola-Kirchhoff stress is given by

$$
\pi=\frac{1}{p} \mathcal{W}^{\prime}(e)
$$

while the back stress is

$$
b:=\frac{1}{p} e \mathcal{W}^{\prime}(e)
$$


The Cauchy stress (denoted here by $\sigma$ ) is given by

$$
\sigma=\pi \text {. }
$$

There is no spin stress (because there are no rotations), thus Clausius-Duhem's inequality (3.9) reduces to

$$
b \dot{p} \geq 0
$$

that is,

$$
e \mathcal{W}^{\prime}(e) \frac{\dot{p}}{p} \geq 0 .
$$

In a one dimensional setting it seems reasonable - to us at least - that the yield criterion act on a quantity that is independent of the plastic strain; this was so in the model developed in Section 3 and it is equally so in Mielke's model (see Remark 4.9). Accordingly, the only possibility is to assume that the relevant stress is $\mathrm{eW}^{\prime}(e)$, admittedly not the Cauchy stress, and then the criterion is

$$
e \mathcal{W}^{\prime}(e) \in \mathbf{K},
$$

where $\mathbf{K}$ is an interval containing 0 . In essence, only a Von-Mises type model may arise in a one-dimensional setting. Assume that $\mathbf{K}$ is of the form $[-c, c], c$ being the yield stress.

The degeneracy of the one-dimensional case allows one to choose either $\dot{p}$, or $\dot{p} / p$ as dual variable in (7.1). Because $p(t)>0$, both settings result in the same elasto-plastic evolution on a segment $(0, L)$, namely,

$$
\begin{array}{r}
e(t), p(t)>0, \varphi^{\prime}(t)=p(t) e(t) \\
\left(\frac{1}{p(t)} \mathcal{W}^{\prime}(e(t))\right)^{\prime}=0 \\
\left|e(t) \mathcal{W}^{\prime}(e(t))\right| \leq c \\
\dot{p}>0 \Rightarrow e(t) \mathcal{W}^{\prime}(e(t))=c \\
\dot{p}<0 \Rightarrow e(t) \mathcal{W}^{\prime}(e(t))=-c
\end{array}
$$

Assume that we further impose the following Dirichlet boundary conditions $\varphi(0)=$ $0, \varphi(L)=L+t d$ (with $d>0$, that is a stretch).

Seeking a variational formulation of the system above in the spirit of Section 4 leads to the following global minimality principle at each time:

Find $e(t), p(t)>0$ such that

$$
\begin{aligned}
\int_{(0, L)} \mathcal{W}(e(t)) d x \leq \int_{(0, L)} \mathcal{W}(\hat{e}) d x+ & \int_{(0, L)} c|\log \hat{p}-\log p(t)| d x, \\
& \forall v^{\prime} \text { with } v(0)=0, v(L)=L+t d, v^{\prime}=\hat{e} \hat{p} .
\end{aligned}
$$

We do not rewrite the energy conservation statement in this framework since it will not be needed in the ensuing analysis.

Note that if, instead of (7.7), we postulated that

$$
\begin{aligned}
\int_{(0, L)} \mathcal{W}(e(t)) d x \leq \int_{(0, L)} \mathcal{W}(\hat{e}) d x+ & \int_{(0, L)} c|\hat{p}-p(t)| d x, \\
& \forall v^{\prime} \text { with } v(0)=0, v(L)=L+t d, v^{\prime}=\hat{e} \hat{p} .
\end{aligned}
$$


we would not obtain the right yield stress condition (7.4), but rather

$$
\left|\frac{1}{p(t)} \mathcal{W}^{\prime}(e(t)) e(t)\right| \leq c .
$$

(The equilibrium equation (7.3) remains unchanged.)

We now propose to study the system (7.2)-(7.6) with associated energy the one which comes out of the variational formulation (7.7), that is

$$
\mathcal{F}(t, \hat{\varphi}, \hat{p}):=\int_{(0, L)} \mathcal{W}(\hat{e}) d x+\int_{(0, L)} c|\log \hat{p}-\log p(t)| d x, \hat{e}:=\frac{\hat{\varphi}^{\prime}}{\hat{p}} .
$$

In truth we do so because we do not know how to identify (energy preserving) global minimizers - or better yet local minimizers - of (7.7), other than through an investigation of the associated system (7.2)-(7.6) which could easily be seen, following the proof of Proposition 4.1, to be satisfied for any smooth solution of the variational evolution.

The free energy $\mathcal{W}$ is taken to be such that

$$
\mathcal{W}(1)=0, \quad\left\{\begin{array}{l}
W \geq 0 \text { strictly convex on its domain } \\
\mathcal{W}(e)=\frac{1}{2} A(e-1)^{2}, e \geq 1, A>0 .
\end{array}\right.
$$

The field $\varphi_{\text {hom }}(x, t):=x(1+t d / L)$ satisfies the boundary condition and is a minimizer as long as $t$ satisfies

$$
\mathcal{W}^{\prime}(1+t d / L)(1+t d / L) \leq c,
$$

that is, as long as $t \leq t_{c}$ where $t_{c}$ is the positive root of

$$
A t^{2} d^{2} / L^{2}+A t d / L-c=0 .
$$

Uniqueness of such a solution-path is not claimed, in contrast with the conclusion that could be drawn in the setting of small strain elasto-plasticity (see e.g. $[9$, Section 5.2]).

Then

$$
\varphi_{\text {hom }}(x, t), \quad e_{\text {hom }}(t)=e_{c}:=1+t_{c} d / L, \quad p_{\text {hom }}(t)=\frac{1+t d / L}{e_{c}}
$$

is a solution for $t \geq t_{c}$.

The solution

$$
\varphi_{\text {hom }}(x, t), e_{\text {hom }}(t)=\left\{\begin{array}{ll}
1+t d / L, & t \leq e_{c} \\
e_{c}, & t \geq t_{c}
\end{array}, p_{\text {hom }}(t)= \begin{cases}1, & t \leq e_{c} \\
\frac{1+t d / L}{e_{c}}, & t \geq t_{c}\end{cases}\right.
$$

is labelled the homogeneous solution. It is indeed the unique spatially homogeneous solution of (7.2)-(7.6) with the boundary conditions $\varphi(0)=0, \varphi(L)=L+t d$.

Remark that it is easily checked that the homogeneous solution of that system is such that, for any smooth pair $(w, \mu)$ with $w=0$ if $x=0, L$, the directional derivative of $\mathcal{F}$ in the smooth direction $(w, \mu)$ satisfies, with obvious notation,

$$
\left\{\begin{array}{l}
D \mathcal{F}\left(\varphi_{\text {hom }}(t), p_{\text {hom }}(t)\right) \cdot(w, \mu) \geq 0 \\
D \mathcal{F}\left(\varphi_{\text {hom }}(t), p_{\text {hom }}(t)\right) \cdot(w, \mu)=0 \quad \text { if } t \geq t_{c}
\end{array}\right.
$$


We investigate the (local) stability properties of the homogeneous solution under smooth perturbations. The notion of stability is interpreted as a statement of nonnegativity of

$$
D^{2} \mathcal{F}\left(\varphi_{\text {hom }}(t), p_{\text {hom }}(t)\right) \cdot[(w, \mu),(w, \mu)]
$$

(the second order directional derivative of $\mathcal{F}$ with, once again, obvious notation) where $\mathcal{F}$ was defined in (7.8), this, provided that the first variation of that energy be 0 . We do so because we view it as intuitive that any finer notion of stability should imply a fortiori stability under smooth variations of $\mathcal{F}$ around the solution $(e(t), p(t))$.

In view of (7.11), stability can only be tested for $t \geq t_{c}$. Take $w$ smooth with $w=0$ if $x=0, L, \mu$ smooth and consider as test $\left(\varphi_{\text {hom }}(t)+h w, p_{\text {hom }}(t)+h \mu\right)$. Then we must have

$$
\begin{aligned}
& \int_{(0, L)}( \frac{1}{2} W^{\prime \prime}\left(\frac{\varphi_{\text {hom }}^{\prime}(t)}{p_{\text {hom }}(t)}\right)\left(\frac{w^{\prime}}{p_{\text {hom }}(t)}-\mu \frac{\varphi_{\text {hom }}^{\prime}(t)}{p_{\text {hom }}^{2}(t)}\right)^{2}+ \\
&\left.W^{\prime}\left(\frac{\varphi_{\text {hom }}^{\prime}(t)}{p_{\text {hom }}(t)}\right)\left(\mu^{2} \frac{\varphi_{\text {hom }}^{\prime}(t)}{p_{\text {hom }}^{3}(t)}-\mu \frac{w^{\prime}(t)}{p_{\text {hom }}^{2}(t)}\right)-c \frac{\mu^{2}}{p_{\text {hom }}^{2}(t)}\right) d x \geq 0 .
\end{aligned}
$$

Take as test functions in (7.12)

$$
\mu \text { smooth with } \int_{0}^{1} \mu(y) d y=0, w(x)=e_{c} \int_{0}^{x} \mu(y) d y .
$$

Then, $w^{\prime}=\mu \varphi_{\text {hom }}^{\prime} / p_{\text {hom }}$, so that (7.12) reduces to

$$
-\frac{c}{p_{\text {hom }}^{2}(t)} \int_{(0, L)} \mu^{2} d x \geq 0
$$

which is a contradiction unless $\mu \equiv 0$. We have established the following proposition

Proposition 7.1. Under assumption (7.9), the unique homogeneous solution ( $\varphi_{\text {hom }}$, $p_{\text {hom }}$ ) of (7.2)-(7.6) with boundary conditions $\varphi(0)=0, \varphi(L)=L+t d$ is unstable under regular perturbations for all $t \geq t_{c}$, that is that there exists a smooth admissible variation $(w, \mu)$ of $\left(\varphi_{\text {hom }}(t), p_{\text {hom }}(t)\right)$ such that

$$
D^{2} \mathcal{F}\left(\varphi_{\text {hom }}(t), p_{\text {hom }}(t)\right)[(w, \mu),(w, \mu)]<0,
$$

with $\mathcal{F}$ defined in $(7.8)$.

In our opinion, the conspiracy between the previous proposition and Lemma 6.1 in Section 6 results in a two-count indictment of the multiplicative decomposition. We, like others, may be misguided in attempting to fit such a model in a variational framework. But it may just be that finite plasticity in the absence of well-defined slip planes does require regularization from the get-go.

Acknowledgements. Both authors are grateful to J.J. MARIGO for his useful advice and many insightful remarks.

GAF wishes to acknowledge the hospitality of the Courant Institute of Mathematical Sciences where his contribution to this work was carried out.

ED warmly thanks the Center for Nonlinear Analysis (NSF Grants No. DMS0405343 and DMS-0635983), where this research was carried out. She was funded under a postdoctoral felloship by the National Science Foundation under Grant No. DMS-0905778. 


\section{REFERENCES}

[1] J. M. Ball and F. Murat. $W^{1, p}$-quasiconvexity and variational problems for multiple integrals. J. Funct. Anal., 58(3):225-253, 1984.

[2] John M. Ball. Some open problems in elasticity. In Geometry, mechanics, and dynamics, pages 3-59. Springer, New York, 2002.

[3] Albrecht Bertram. Elasticity and plasticity of large deformations. Springer, Heidelberg, third edition, 2012. An introduction.

[4] B. Bourdin, G. A. Francfort, and J.-J. Marigo. The variational approach to fracture. Springer, New York, 2008. Reprinted from J. Elasticity 91 (2008), no. 1-3 [MR2390547], With a foreword by Roger Fosdick.

[5] S. Conti, G. Dolzmann, and C. Kreisbeck. Asymptotic behavior of crystal plasticity with one slip system in the limit of rigid elasticity. SIAM J. Math. Anal., 43(5):2337-2353, 2011.

[6] S. Conti, G. Dolzmann, and C. Kreisbeck. Relaxation and microstructure in a model for finite crystal plasticity with one slip system in three dimensions. Discrete Contin. Dyn. Syst. Ser. $S, 6(1): 1-16,2013$.

[7] S. Conti and F. Theil. Single-slip elastoplastic microstructures. Arch. Ration. Mech. Anal., 178(1):125-148, 2005.

[8] Walter J. Culver. On the existence and uniqueness of the real logarithm of a matrix. Proc. Amer. Math. Soc., 17:1146-1151, 1966.

[9] G. Dal Maso, A. DeSimone, and M. G. Mora. Quasistatic evolution problems for linearly elastic-perfectly plastic materials. Arch. Ration. Mech. Anal., 180(2):237-291, 2006.

[10] G. Dal Maso, A. DeSimone, and F. Solombrino. Quasistatic evolution for Cam-Clay plasticity: a weak formulation via viscoplastic regularization and time rescaling. Calc. Var. Partial Differential Equations, 40(1-2):125-181, 2011.

[11] G. Dal Maso, G. A. Francfort, and R. Toader. Quasistatic crack growth in nonlinear elasticity. Arch. Ration. Mech. An., 176(2):165-225, 2005.

[12] G. Dal Maso and R. Toader. A model for the quasi-static growth of brittle fractures: Existence and approximation results. Arch. Rational Mech. Anal., 162:101-135, 2002.

[13] N.A. Fleck and J.W. Hutchinson. Strain gradient plasticity. Adv. Appl. Mech., 33:295-361, 1997.

[14] N.A. Fleck and J.W. Hutchinson. A reformulation of strain gradient plasticity. J. Mech. Phys Solids, 49(10):2245-2271, 2001.

[15] G.A. Francfort and A. Garroni. A variational view of partial brittle damage evolution. Arch. Rational Mech. Anal., 182(1):125-152, 2006.

[16] G.A. Francfort and A. Giacomini. Small-strain heterogeneous elastoplasticity revisited. Comm. Pure Appl. Math., 65(9):1185-1241, 2012.

[17] G.A. Francfort and C. Larsen. Existence and convergence for quasi-static evolution in brittle fracture. Comm. Pure Appl. Math., 56(10):1465-1500, 2003.

[18] G.A. Francfort and J.-J. Marigo. Revisiting brittle fracture as an energy minimization problem. J. Mech. Phys. Solids, 46(8):1319-1342, 1998.

[19] G.A. Francfort and J.J. Marigo. Stable damage evolution in a brittle continuous medium. Eur. J. Mech. A-Solid, 12(2):149-189, 1993.

[20] G.A. Francfort and A. Mielke. Existence results for a class of rate-independent material models with nonconvex elastic energies. J. reine angew. Math., 595:55-91, 2006.

[21] M. Gurtin, E. Fried, and L. Anand. The Mechanics and Thermodynamics of Continua. Cambridge University Press, Cambridge, 2010.

[22] B. Halphen and Q.S. Nguyen. Sur les matériaux standards généralisés. J. Mec., 14(1):39-63, 1975.

[23] Vlado Lubarda. Elastoplasticity theory. CRC Press, Boca Raton, FL, 2001.

[24] Jacob Lubliner. Plasticity Theory. Macmillan Publishing, New York, 1990.

[25] A. Mainik and A. Mielke. Existence results for energetic models for rate-independent systems. Calc. Var. Partial Differential Equations, 22(1):73-99, 2005.

[26] A. Mainik and A. Mielke. Global existence for rate-independent gradient plasticity at finite strain. J. Nonlinear Sci., 19(3):221-248, 2009.

[27] J.E. Marsden and T.J.R. Hughes. Mathematical foundations of elasticity. Dover Publications Inc., New York, 1994. Corrected reprint of the 1983 original. 
[28] A. Mielke and T. Roubíček. Rate-independent damage processes in nonlinear elasticity. Math. Models Methods Appl. Sci., 16(2):177-209, 2006.

[29] A. Mielke and U. Stefanelli. Linearized plasticity is the evolutionary $\gamma$-limit of finite plasticity. J. Eur. Math. Soc., 15(3):923-948, 2013.

[30] Alexander Mielke. Finite elastoplasticity, lie groups and geodesics on sl(d). In A. Weinstein P. Newton and P.J. Holmes, editors, Geometry, Dynamics, and Mechanics, pages 61-90. Springer-Verlag, 2002.

[31] Alexander Mielke. Energetic formulation of multiplicative elasto-plasticity using dissipation distances. Contin. Mech. Thermodyn., 15(4):351-382, 2003.

[32] Alexander Mielke. Existence of minimizers in incremental elasto-plasticity with finite strains. SIAM J. Math. Anal., 36(2):384-404 (electronic), 2004.

[33] Alexander Mielke. Evolution of rate-independent systems. In A. Dafermos and E. Feireisl, editors, Evolutionary equations. Vol. II, Handb. Differ. Equ., pages 461-559. Elsevier/NorthHolland, Amsterdam, 2005.

[34] F. Murat and C. Trombetti. A chain rule formula for the composition of a vector-valued function by a piecewise smooth function. Boll. Unione Mat. Ital. Sez. B Artic. Ric. Mat. (8), 6(3):581-595, 2003.

[35] Paul M. Naghdi. A critical review of the state of finite plasticity. Z. Angew. Math. Phys., 41(3):315-394, 1990.

[36] Miroslav Šilhavý. The mechanics and thermodynamics of continuous media. Texts and Monographs in Physics. Springer-Verlag, Berlin, 1997.

[37] Luc Tartar. Compensated compactness and applications to partial differential equations. In Nonlinear analysis and mechanics: Heriot-Watt Symposium, Vol. IV, volume 39 of Res. Notes in Math., pages 136-212. Pitman, Boston, Mass., 1979.

[38] Clifford Truesdell. Rational thermodynamics. Springer-Verlag, New York, second edition, 1984. With an appendix by C. C. Wang, With additional appendices by 23 contributors.

(Elisa Davoli) Department of Mathematics, Center for Nonlinear Analysis, Carnegie Mellon University

E-mail address, E. Davoli: edavoli@andrew.cmu.edu

(Gilles Francfort) LAGA, Université Paris-Nord \& Institut Universitaire de France

E-mail address, G. Francfort: gilles.francfort@univ-paris13.fr 